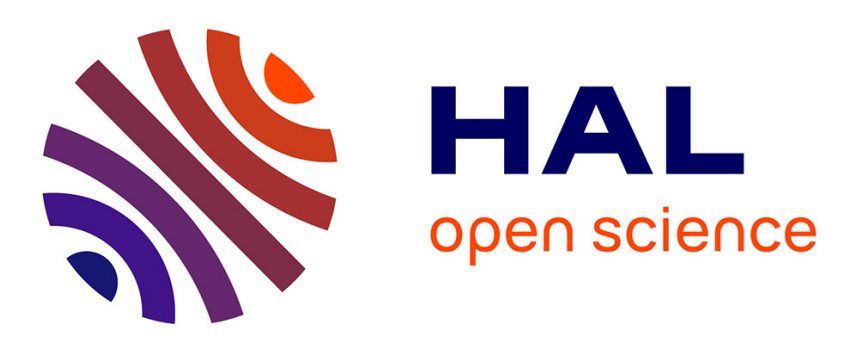

\title{
Nonlinear transient vibrations and coexistences of multi-instabilities induced by friction in an aircraft braking system
}

Fabrice Chevillot, Jean-Jacques Sinou, Nicolas Hardouin

\section{- To cite this version:}

Fabrice Chevillot, Jean-Jacques Sinou, Nicolas Hardouin. Nonlinear transient vibrations and coexistences of multi-instabilities induced by friction in an aircraft braking system. Journal of Sound and Vibration, 2009, 328 (4-5), pp.555-574. 10.1016/j.jsv.2009.08.028 . hal-00425155

\section{HAL Id: hal-00425155 \\ https://hal.science/hal-00425155}

Submitted on 25 Sep 2012

HAL is a multi-disciplinary open access archive for the deposit and dissemination of scientific research documents, whether they are published or not. The documents may come from teaching and research institutions in France or abroad, or from public or private research centers.
L'archive ouverte pluridisciplinaire HAL, est destinée au dépôt et à la diffusion de documents scientifiques de niveau recherche, publiés ou non, émanant des établissements d'enseignement et de recherche français ou étrangers, des laboratoires publics ou privés. 


\title{
Nonlinear transient vibrations and coexistences of multi instabilities induced by friction in an aircraft braking system
}

\author{
F. Chevillot ${ }^{\mathrm{a}, \mathrm{b}}$, J.-J. Sinou ${ }^{*, a}$, N. Hardouin ${ }^{\mathrm{b}}$ \\ ${ }^{a}$ Laboratoire de Tribologie et Dynamique des Systèmes, UMR CNRS 5513, \\ École Centrale de Lyon, 69134 Ecully, France \\ ${ }^{b}$ Messier-Bugatti, Aircraft Braking Division, Zone Aéronautique Louis Bréguet, \\ BP 40, 78140 Vélizy-Villacoublay, France
}

\begin{abstract}
Friction-induced vibration is still a cause for concern in a wide variety of mechanical systems, because it can lead to structural damage if high vibration levels are reached. Another effect is the noise produced that can be very unpleasant for end-users, thereby making it a major problem in the field of terrestrial transport. In this work the case of an aircraft braking system is examined. An analytical model with polynomial nonlinearity in the contact between rotors and stators is considered.

Stability analysis is commonly used to evaluate the capacity of a nonlinear system to generate frictioninduced vibrations. With this approach, the effects of variations in the system parameters on stability can be easily estimated. However, this technique does not give the amplitude of the vibrations produced. The integration of the full set of nonlinear dynamic equations allows computing the time-history response of the system when vibration occurs. This technique, which can be time-consuming for a model with a large number of degrees of freedom (dof), is nevertheless necessary in order to calculate the transient-state behavior of the system. The use of a Continuous Wavelet Transform (CWT) is very suitable for the detailed analysis of the transient response. In this paper, the possibilities of coexistence of several instabilities at the same time will be examined. It will be shown that the behavior of the brake can be very complex and cannot be predicted by stability analysis alone.
\end{abstract}

Key words: friction-induced vibration, transient oscillations, continuous wavelet transform, damping

\section{Introduction}

By nature, brakes in the aeronautical, automotive and rail industries are systems that comprise rubbing interfaces. A major concern in such systems is the emergence of vibrations induced by friction. Although it has been the subject of many investigations over recent decades, friction-induced instabilities are still an active field of research in dynamics. Manufacturers suffer from a lack of solutions allowing them to eliminate or reduce such vibrations.

Many mechanisms have been proposed to explain the emergence of friction-induced instabilities. First, Spurr [1] proposed a sprag-slip phenomenon based on geometrically induced instability. With the works of Jarvis and Mills [2], his theory was expanded into a more generic coupling of degrees of freedom. Most recent studies on friction-induced instabilities have been performed on the basis of this theory. However, another approach was proposed at the same time and is still under investigation: in 1938, Mills [3] and later Fosberry and Holubecki [4] and Ouyang et al. [5] considered the decrease in the friction coefficient with relative sliding speed as a source of destabilization. Lastly, the mechanism known as stick-slip (Antoniou

\footnotetext{
${ }^{*}$ Corresponding author

Email address: jean-jacques.sinou@ec-lyon.fr (J.-J. Sinou)

Preprint submitted to Journal of Sound and Vibration
}

September 25, 2012 
et al. [6]) can be used to explain the emergence of vibrations. This mechanism is caused by the static friction coefficient becoming higher than the dynamic coefficient. This theory is adapted to low sliding velocities, which allow the alternation of sliding and sticking states. Finally, the four mechanisms considered responsible for friction-induced vibrations fall into two main categories. On the one hand, stick-slip and a decreasing friction coefficient with sliding speed imply variations in the friction coefficient. Thus they are more specifically a question of tribological properties. With an approach involving sprag-slip or a geometric coupling of degrees of freedom, instability can occur even if the friction coefficient is constant.

In this paper an aircraft braking system is studied. Lyu et al. [7, 8] and Gordon [9] were among the first to present the equations of motion and a model description to simulate the squeal phenomenon in aircraft braking systems. In their approach, instability is the result of geometric coupling between lateral translation and the yaw of the rotors and stators. The model predicts that instability can occur with a constant coefficient of friction and without the use of a negative damping. Studies on whirl, another kind of friction-induced instability in aircraft brakes, were first performed by Travis [10] and Hagler [11], and completed by the work of Sinou et al. $[12,13]$ by using geometric coupling and sprag-slip approaches. Finally, a complete nonlinear approach for whirl and squeal instabilities has been presented by Chevillot et al. [14].

A stability analysis is commonly performed to evaluate the stability of the system by calculating the complex eigenvalues of the Jacobian matrix of the system of equations linearized at the equilibrium point. The study of the eigenvalues allows determining whether a given configuration of the system can generate oscillations. The nature of the instabilities can also be determined, their frequencies calculated and their analytical mode shape plotted. To stabilize the system, work can be done during the design in order to identify a set of parameters for which stability analysis does not reveal any instability. However, such a configuration is very hard to determine in practice. Consequently, other approaches have to be envisaged in a context in which the system cannot be easily stabilized. The limits of the stability analysis are that no information is provided about the amplitude of the oscillations that the mechanism will generate. Nevertheless, the real design criterion concerns the amplitude of the vibration: an instability may lead to very small oscillations in the time-history response just as it may lead to very large oscillations. Thus the dynamical behavior of the system has to be determined to complete the preliminary results of the stability analysis. It can then be very useful to calculate the amplitude of the limit-cycles. To achieve this, numerical methods have been proposed to compute the steady-state oscillations without integrating the full nonlinear equations of motion, for example, the Constrained Harmonic Balance Method [15]. However, experimental tests on an aircraft braking system (one example can be found in [12]) show that determining the stationary regime alone can be misleading. Firstly, transient oscillations may occur with a much higher amplitude than that of the steady-state oscillations; secondly, instabilities may develop in the transient response but only for a short duration, though they are not present in the steady-sate response. Thus it is difficult to avoid full integration of the nonlinear system in order to obtain the time-history response. Whereas the computation of stability and steady-state oscillations is fast, temporal integration requires particular attention. Finite element models with a large number of degrees of freedom can be used (Abubakar and Ouyang [16], Massi et al. [17] for instance), but in spite of numerical efforts, computation time is still an issue. The use of analytical models with a minimum number of degrees of freedom could be be a way of getting round this obstacle.

The first part of this paper presents an aircraft braking system and describes the analytical model built to simulate friction-induced instabilities. After this a brief description is given of the methods employed for time-frequency analysis, in particular the Continuous Wavelet Transform. The stability analysis is then performed in order to determine the nature of the instabilities and the evolution of the stability with respect to the coefficient of friction. In the last section, time-history responses are computed and the nonlinear dynamical transient and steady-state behaviors are analyzed with the help of CWT. Changes in the damping configuration are made without concern given to the physical evaluation of the real damping of the structure, in order to produce complex responses involving several instabilities. 


\section{Description and nonlinear modeling of the brake system}

\subsection{Description of an aircraft braking system}

Fig. 1 shows a schematic model of an aircraft braking system where the rotating parts are given in a three-quarter section view. The brake is attached to the landing gear of the aircraft through a torque take-out rod. The core of the brake is composed of a stack of rotating and stationary discs whose number depends on the size of the aircraft: the rotating discs -the rotors- are engaged by the wheel, while the stationary discs -the stators- are linked to the torque tube which is interdependent of the piston housing. During braking, the rotors and stators are squeezed together by pressure in the hydraulic pistons. Torque is produced by friction forces generated at the rubbing interfaces between the discs. Aircraft slowing and stopping is therefore the result of the conversion of the kinetic energy of the plane into heat in the brake.

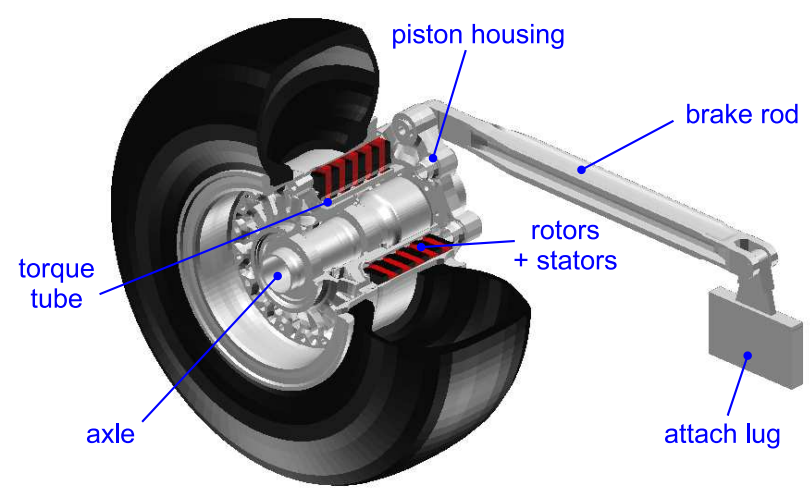

Figure 1: Schematic model of the brake system

The evaluation of the dynamical behavior of the brake under working conditions is performed with the help of dynamic tests using a fully instrumented aircraft brake (for a more detailed view of the instrumentation see [12]). Two main complex nonlinear vibrations are then identified: squeal and whirl. Both appear at low frequency (in the $0-1,000 \mathrm{~Hz}$ range) with sometimes large amplitude oscillations that liable to damage the integrity of the brake. In reality, here is no single squeal and whirl vibration, as these terms define more generic vibratory phenomena defined in [7], whose deformations differ. On the one hand, squeal is defined as torsional vibrations of nonrotating brake parts around the axle; on the other hand, whirl describes a motion of the end of the torque tube around the axle acompanied by unphased pumping of the brake pistons. An example of two analytical mode shapes, one of each kind, is presented in section 4 .

\subsection{Nonlinear modeling of the brake system}

\subsubsection{Description of the 70 degree-of-freedom model}

The nonlinear analytical model built to reproduce whirl and squeal instabilities is represented in Fig. 2. The brake, with the brake rod and attachement lug, is modeled by writing the equations of motion with MATLAB ${ }^{\mathrm{TM}}$. With 70 degrees of freedom, good accuracy is expected in the simulation of instabilities in the 0-1,000 $\mathrm{Hz}$ range. A reduced number of degrees of freedom is a susbstantial advantage for the full temporal integration of the dynamical equations. This model has already been used by Chevillot et al. [14] to study the effects of damping on stability.

\subsubsection{Nonlinear contact stiffness}

Experimental tests [12] are conducted in order to record the behavior of the disc stack in compression. As suggested by Sinou et al.[12], the nonlinear load-compression relationship is introduced analytically by 


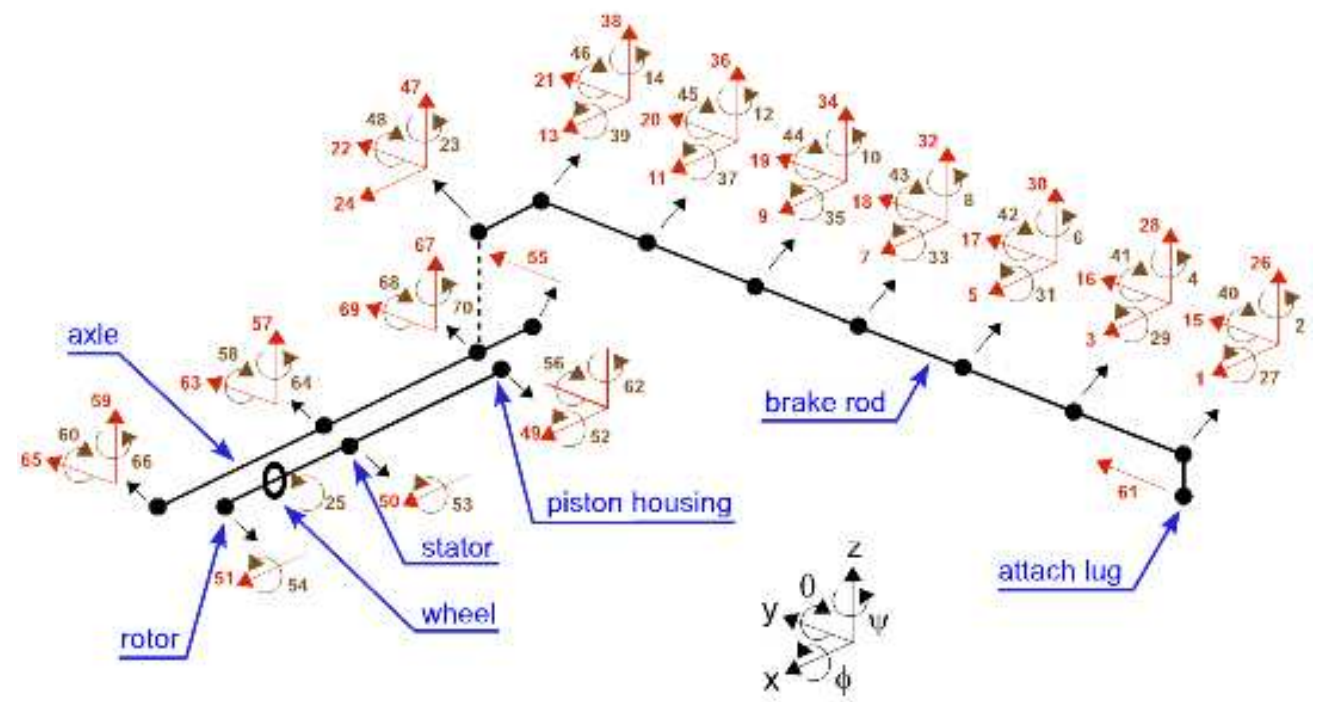

Figure 2: 70 degree-of-freedom analytical model

a third order polynomial in the relative displacement between the discs (for reasons of confidentiality the values of the $K_{i}$ coefficients cannot be given).

$$
P=\sum_{i=1}^{3} K_{i} \delta x^{i}
$$

with $K_{i}$ (i=1 to 3 ) the coefficients of the $3^{\text {rd }}$ order polynomial.

\subsubsection{Friction modeling}

The multistage brake is represented by a single rotor and stator with an effective brake friction coefficient $\mu_{e q}=2 N_{\text {rotor }} \mu$ where $N_{\text {rotor }}$ is the number of rotors (leading to $2 N_{\text {rotors }}$ interfaces in contact between the stators and rotors). It is assumed that each rubbing interface (a ring of inner radius $R_{i}$ and outer radius $R_{e}$ as illustraed in Fig. 3) is equivalent and that the friction coefficient is uniform on the surface. Moreover, contact and sliding states are assumed to be permanent.
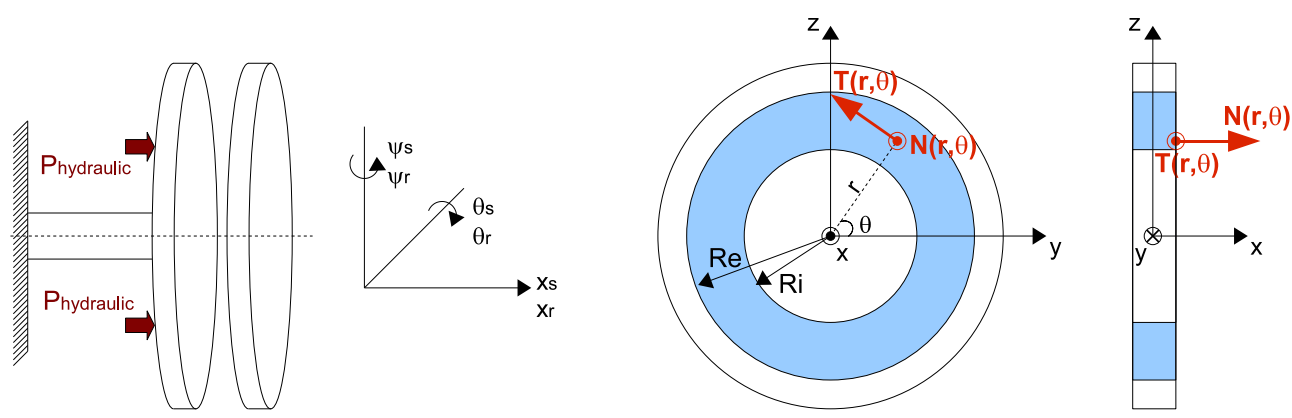

stator rotor

Figure 3: Friction modeling at the stator-rotor interface

The friction forces are deduced from the contact forces by using the classical Coulomb law: the tangential stress $T$ is generated by the coefficient of friction: 


$$
\begin{gathered}
N(r, \theta)=P(r, \theta) \\
T(r, \theta)=\mu_{e q} N(r, \theta)=\mu_{e q} P(r, \theta)
\end{gathered}
$$

Considering the nonlinear behavior of the normal stress acting at the interface between the discs (Eq. (1)), and the relationship between the normal stress and the tangential stress (Eqs. (2) and (3)), the global expression of the nonlinear terms $\mathbf{F}_{\mathrm{NL}}$ can be expressed. The latter have been presented in a previous study [14] and are developed in Appendix A. After calculation, the nonlinear equations of motions can be written in the following form [14]:

$$
\mathbf{M} \ddot{\mathbf{x}}+\mathbf{C} \dot{\mathbf{x}}+\mathbf{K x}=\mathbf{F}_{\mathrm{NL}}(\mathbf{x})+\mathbf{F}_{\text {pressure }}+\mathbf{F}_{\text {tyreload }}
$$

where $\ddot{\mathbf{x}}, \dot{\mathbf{x}}$ and $\mathbf{x}$ are respectively the 70 -dimensional vectors of acceleration, velocity and displacement. $\mathbf{M}, \mathbf{K}$ and $\mathbf{C}$ are respectively the structural mass, stiffness and damping matrices. $\mathbf{F}_{\text {pressure }}$ is the vector force due to brake hydraulic pressure, $\mathbf{F}_{\mathrm{NL}}$ contains the linear and non-linear contact force terms at the stator and rotor interface, and $\mathbf{F}_{\text {tyreload }}$ is the bending load applied on the tyre.

\subsubsection{Damping modeling}

The damping matrix $\mathbf{C}$ mentioned in Eq. (4) is built with a modal approach. An elementary modal damping matrix $\mathbf{D}_{i}$ is specified for the i-th mode of the structure, of angular frequency $\omega_{i}$, by its modal damping coefficient $\eta_{i}$ :

$$
\mathbf{D}_{i}=2 \eta_{i} \omega_{i} \mathbf{E}_{i}
$$

where $\mathbf{E}_{i}$ is the elementary matrix with only 1 on the i-th row and i-th column

A global modal damping matrix is then expressed by:

$$
\mathbf{D}=\sum_{i=1}^{70} \mathbf{D}_{i}
$$

The structural damping matrix $\mathbf{C}$ is then constructed by projecting the modal damping matrix $\mathbf{D}$ onto the undamped, non-frictional inverse modal basis $\boldsymbol{\Phi}^{-1}$ :

$$
\mathbf{C}=\boldsymbol{\Phi}^{-1^{T}} \mathbf{D} \boldsymbol{\Phi}^{-1}
$$

In comparison with proportional damping, often considered in numerical studies due to its simplicity, the great advantage of this approach is the independence of each mode with regard to damping. This is especially helpful for the study because each mode, and therefore each instability, can be controlled separately with the modification of its modal damping coefficient.

\section{Methods for time-frequency analysis}

The frequency analysis is a key step in the investigation of the information contained in a given signal. For a stationary signal, where time does not play a role, the calculation of the frequency components of the whole signal is sufficient. The Fast Fourier Transform (FFT) is therefore a commonly used method for studying stationary signals: it transforms the signal from a time-based domain to a frequency-based domain. In the case of a nonstationary signal, the time-dependence of the frequency components can be an essential point to evaluate in order to obtain good understanding of the behavior. To achieve this, methods are employed to generate time-frequency representations of the signal. For a nonstationary signal with sharp time-varying frequency components, the performance and precision of the method employed are determining elements. 


\subsection{The Short-Time Fourier Transform}

The Short-Time Fourier Transform (STFT), first proposed by D. Gabor in [18], is a commonly used method for time-frequency analysis, since it is a direct extension of the Fourier transform. Only a small section of the signal is analyzed at a time through a window function. The division of the signal into sections allows generating a time-frequency representation of the signal.

In the case of a weak nonstationary signal $s(t)$, the section of the signal of duration $T$ centered at time location $\tau$ is assumed to be stationary when seen through a window $w(t)$. The STFT at location $\tau$ is the Fourier transform of the windowed signal $s(t) w(t-\tau)$ :

$$
\operatorname{STFT}_{\tau}^{s}(f)=\int_{-T / 2}^{T / 2} s(t) w(t-\tau) e^{-j 2 \pi f t}
$$

The drawback of STFT is that the information on time and frequency can be obtained with only limited precision determined by the size $T$ of the window. Thus the analysis suffers from the dilemma of resolution: with a narrow window, only poor frequency resolution can be obtained but with good time localization; on the other hand, good frequency resolution can be obtained with a wide window, but at the expense of time localization. Moreover, the choice of the window function itself may have an effect on estimation accuracy. Consequently, the STFT method can be employed in the case of signals with weak nonstationary characteristics that allow using a fairly large window size and obtaining satisfactory accuracy.

\subsection{The Continuous Wavelet Transform}

To accurately analyze a signal with pronounced nonstationary behavior, the Continuous Wavelet Transform (CWT) can be used. Wavelet transforms are based on time-scale methods that give better timefrequency resolution than the standard STFT. They first appeared in the 1980s with the work of J. Morlet [19]. The theoretical background can be found in [20,21, 22], though only the main principles are stated in the following section.

A wavelet analysis starts by selecting an elementary wavelet function $\phi(t)$ whose decay is very fast and which fulfills the admissibility conditions [21, 22]: the mother wavelet. A family of functions (the wavelets) are then obtained by translation and dilatation of the mother wavelet:

$$
\phi_{a, b}(t)=\frac{1}{\sqrt{a}} \phi\left(\frac{t-b}{a}\right)
$$

where $a$ is the dilatation or scale factor and $b$ is the time translation factor. The term $1 / \sqrt{a}$ ensures the energy normalization across the different scales.

The transform is then performed by projecting the signal $s(t)$ onto the wavelets, producing the CWT coefficients:

$$
C W T_{\phi}^{s}(a, b)=\int_{-\infty}^{\infty} s(t) \phi_{a, b}^{*}(t) d t=\frac{1}{\sqrt{a}} \int_{-\infty}^{\infty} s(t) \phi^{*}\left(\frac{t-b}{a}\right) d t
$$

where ${ }^{*}$ denotes the complex conjugate.

The wavelet coefficients represent a measurement of the correlation of the dilated and shifted wavelet with the function $s(t)$. $C W T_{\phi}^{s}(a, b)$ also express the local information on $s(t)$ at time $t=b$ and frequency $f$ related to $1 / a$. A time-frequency representation is obtained by plotting the wavelet coefficients over time

and frequency. In practice, it is preferable to plot the wavelet power spectrum, defined as $\left|C W T_{\phi}^{s}(f, b)\right|^{2}$, because of its analogy with the Fourier power spectrum [23]. A 3-D graph can be drawn, but the CWT of a signal is usually drawn in the form of a 2-D graph with the levels represented by a $\log 2$ color scale.

Many types of wavelets with their own features and performance in time and frequency domains have been developed. One of the most widely used mother wavelets is the Morlet wavelet defined as:

$$
\phi(t)=e^{j k_{0} t} e^{-\frac{1}{2} t^{2}} \stackrel{F T}{\longrightarrow} \hat{\phi}(\omega)=\sqrt{2 \pi} e^{-\frac{1}{2}\left(\omega-k_{0}\right)^{2}}
$$


In the case of the Morlet wavelet, the relation between scale and frequency is given by the expression of the central frequency $\omega_{c}=k_{0} / a$ at which $\hat{\phi}(a \omega)$ is maximal [24]. The factor $k_{0}$ is called the wavenumber. Since $\hat{\phi}(0) \neq 0$, the Morlet wavelet given here does not scrupulously respect the admissibility condition imposed on a wavelet, which is necessary to obtain the inverse of the transform [22]. A correction factor can be applied, but for a wavenumber higher than 6 the error may be considered negligible. Moreover, the inverse transform will not be used in this study. In practice, the wavenumber is a useful parameter because it allows a trade-off between time and frequency resolutions: the higher the wavenumber the better the frequency accuracy, but at the expense of temporal accuracy.

The CWT uses a short window at high frequencies and a long window at low frequencies, in contrast to the FFT which uses a single analysis window. This partially overcomes the time-resolution limitation of the STFT.

\section{Stability analysis}

The stability analysis is the first step for studying nonlinear systems subjected to instability phenomena. It allows determining the nature of the instabilities possibly generated by the system: frequency, mode shape, stability area. The stability analysis is conducted around the equilibrium point $\mathbf{x}_{0}$ computed for a given set of parameters when pressure is applied. It is given by solving the nonlinear system defined by Eq. (4) in the static case:

$$
\mathbf{K}_{0}=\mathbf{F}_{\mathrm{NL}}\left(\mathbf{x}_{0}\right)+\mathbf{F}_{\text {pressure }}+\mathbf{F}_{\text {tyreload }}
$$

The stability is then investigated around the equilibrium point for a small perturbation $\overline{\mathbf{x}}$ by linearizing the nonlinear contact forces:

$$
\mathbf{M} \ddot{\overline{\mathbf{x}}}+\mathbf{C} \dot{\overline{\mathbf{x}}}+\left(\mathbf{K}-\left.\frac{\partial \mathbf{F}_{\mathrm{NL}}}{\partial \overline{\mathbf{x}}}\right|_{\mathbf{x}_{0}}\right) \overline{\mathbf{x}}=\mathbf{0}
$$

The previous equation is written in the state-space $[\overline{\mathbf{x}}, \dot{\overline{\mathbf{x}}}]^{T}$ that gives the Jacobian matrix:

$$
\mathbf{J}=\left[\begin{array}{cc}
\mathbf{0} & \mathbf{I} \\
-\mathbf{M}^{-1}\left(\mathbf{K}-\left.\frac{\partial \mathbf{F}_{\mathrm{NL}}}{\partial \mathbf{x}}\right|_{\mathbf{x}_{0}}\right) & -\mathbf{M}^{-1} \mathbf{C}
\end{array}\right]
$$

For $\lambda$ a complex eigenvalue of $\mathbf{J}$ expressed as $\lambda=a+i \omega$, the stability criterion is: if $a$ is negative or zero, the system is stable and no vibration occurs, and if $a$ is positive then the system is unstable. The imaginary part $\omega$ represents the angular frequency of the vibration. In addition, a damping coefficient can be expressed by $\eta=-a /|a+i \omega|$ (if the system is unstable then at least one of the damping coefficients is strictly negative). The plots of the frequencies (the angular frequencies divided by $2 \pi$ ) and of the damping coefficients versus the brake friction coefficient are given respectively in Fig. 4(a) and (b). No structural damping is added for this calculation (for more details about the effects of damping on stability see [14, 25, 26, 27, 28, 29]). Five instabilities are calculated between 0 and $800 \mathrm{~Hz}$, corresponding to the frequency range in which the analytical model is assumed to be efficient. They are plotted by bold red lines in the figures. Each instability is the result of the coupling by the friction of two modes of the structure. The nature of the instabilities can be identified by plotting the analytical mode shapes: three of them turn out to be whirl instabilities, while two have squeal characteristics. One mode shape of each kind is given in Fig. 5 to illustrate the differences between the two kinds of instabilities. The shaded lines define the static position of the brake.

Fig. 4(a) shows that increasing the friction coefficient causes the Squeal 1 instability to disappear. Indeed, the coalescence of the two modes is not permanent: the two modes merge when reaching the first Hopf bifurcation point, but a second Hopf bifurcation point exists for larger values of the driving parameter (in this case the coefficient of friction) above which the modes are no longer merged. This means that if the stability graphs of Fig. 4(a) and (b) were given for larger values of the coefficient of friction, all five instabilities could be seen to disappear. For example, for the Whirl 2 instability, the decrease of the damping coefficient in Fig. 4(b) can be noted: when reaching zero, the second bifurcation Hopf is reached and the 
(a)

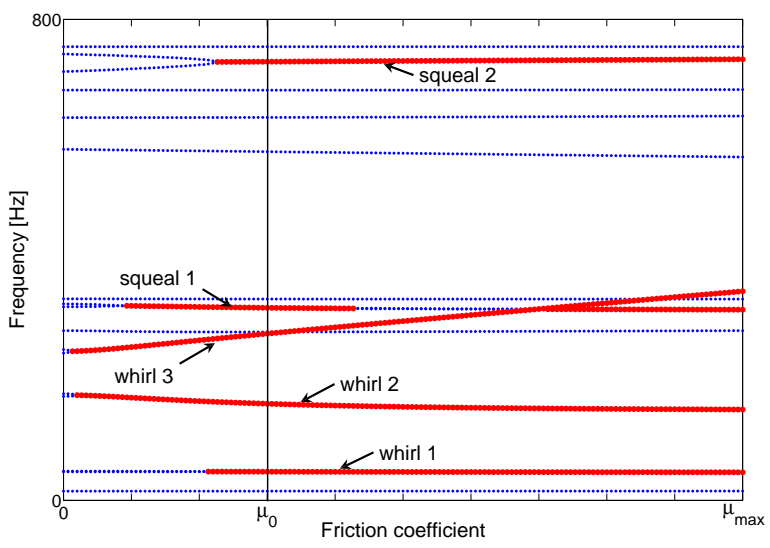

(b)

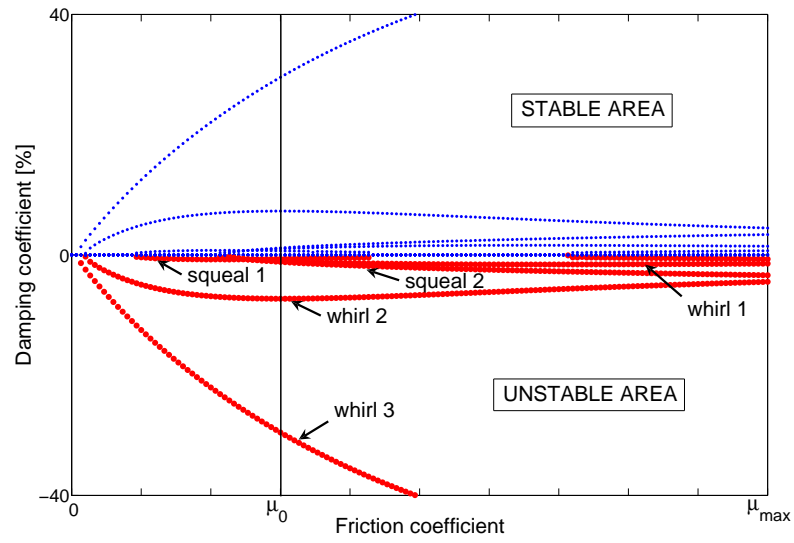

Figure 4: Stability wrt the friction coefficient: (a) frequencies, (b) damping coefficients

(a)



(b)

(1)

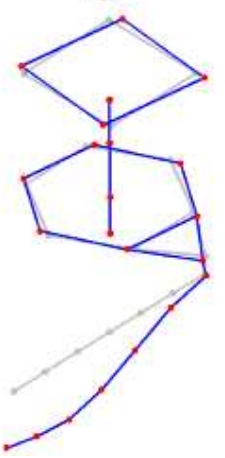

(2)

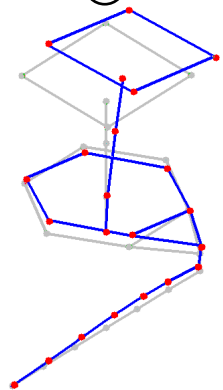

(2)

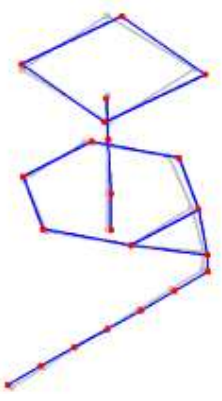

(3)

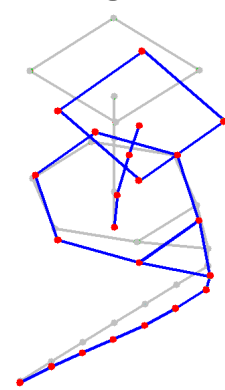

(3)

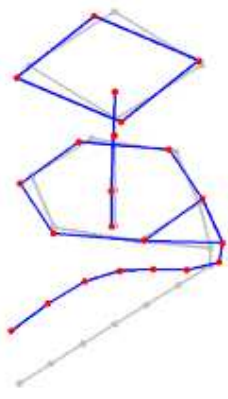

(4)

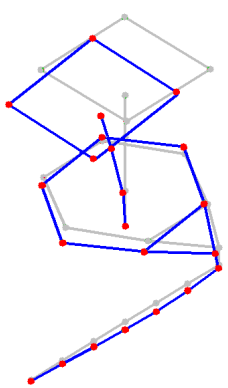

(4)



(5)

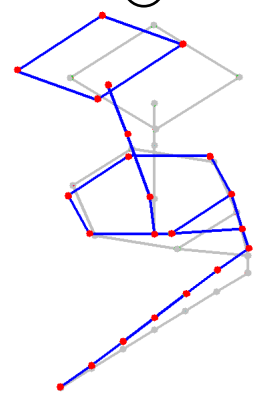

(5)

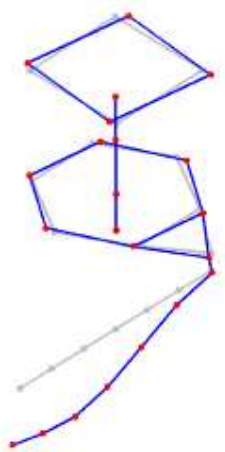

Description:

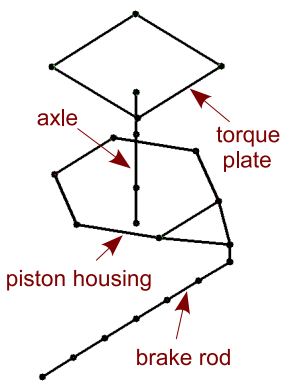

Description:

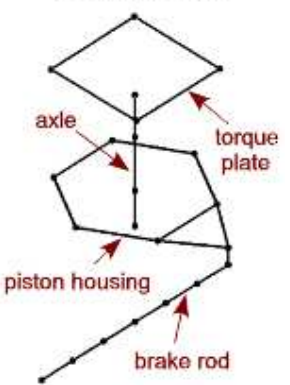

Figure 5: Analytical mode shapes: (a) Whirl 1 instability, (b) Squeal 1 instability 
instability disappears. More information about mode-coupling instabilities and Hopf bifurcation points can be found, for instance, in $[12,13]$.

The configuration for a brake friction coefficient of $\mu_{0}$ marked in Fig. 4 appears very interesting because the five instabilities are destabilized at the same time. Therefore the choice of this friction coefficient is perfectly suited for the evaluation of multi-instability behaviors, even if it does not correspond exactly to a real value of the brake friction coefficient. Thus, the stability analysis has allowed us to identify the instabilities possibly generated by the brake and choose an interesting value of the friction coefficient. Timehistory simulations are then computed in order to identify the temporal behavior of the brake in vibration. They are presented in the following parts of this paper.

\section{Nonlinear analysis with the use of CWT}

Determining the stability of the brake is useful as a preliminary study but does not give the amplitude oscillations or the transient behavior of the brake. To do this, the nonlinear equations of motion have to be integrated numerically to obtain the time-history response. Several simulations are performed when one or more instabilities occur. The brake is first placed under steady-state operating conditions, determined by the calculation of the equilibrium point of the system when pressure is applied. The dynamical behaviour is then investigated around the static position of the system. All the parameters (pressure, coefficient of friction, damping) remain constant throughout the simulation. The continuous wavelet transform (CWT) based on the Morlet mother wavelet as defined in Section 3.2 is used to study the time-history responses. The analysis is performed for two specific degrees of freedom that may be located in Fig. 2: dof 21 which represents the traction/compression of the torque take-out rod, and dof 49 which characterizes the axial displacement of the piston housing.

The previous stability analysis allowed highlighting a set of parameters for which five coupling instabilities are calculated. As specified no damping was used for this calculation. For the time-history simulations, the modal damping defined in Section 2.2.4 is added in the equations of motion. The modal approach allows considering each instability separately: for the friction coefficient $\mu_{0}$ determined in the previous section, in order to obtain a configuration without a specific instability, enough damping on the two modes producing this instability can be added to prevent it from developing. In this way, all the configurations with $1,2,3$, 4 or 5 instabilities can be obtained. The damping configuration for each simulation performed is given in Appendix B.

\subsection{Single instability behavior}

Simulations are conducted in order to study the behavior of the five coupling instabilities taken separately. They are presented in ascending order of complexity.

\subsubsection{Squeal 2}

The first case presented is the time-history response of the brake when a Squeal 2 instability occurs. The temporal integration between 0 and 3 seconds is given in Fig. 6 for dof 21 (a) and dof 49 (b). The dynamics of the response is simple and is composed of two steps: in the first step, the amplitude oscillation increases exponentially, then in the second step the oscillations stabilize and limit-cycle amplitude is reached. In particular, no overshoot in the transient regime is observed. It should be noted that the instability develops quite slowly since the steady-state oscillations are reached after around 2.1 seconds.

An FFT is performed to calculate, with the approximation of stationary signals, the frequencies occurring in the dynamical response. The power spectrum reveals that the response is made up of a single frequency calculated at around $724 \mathrm{~Hz}$. This frequency may be compared with that of around $730 \mathrm{~Hz}$ obtained by the previous stability analysis. Although the stability calculation was performed without damping, the addition of damping cannot be the cause for the gap in frequency: if the stability analysis is conducted with damping, the frequency of the instability only decreases from $729.75 \mathrm{~Hz}$ to $729.57 \mathrm{~Hz}$. In fact, the stability analysis is performed with a linear approximation at the equilibrium point, while the temporal response integrates the full nonlinear equations, giving rise to the slight difference in frequencies noticed. 
(a)
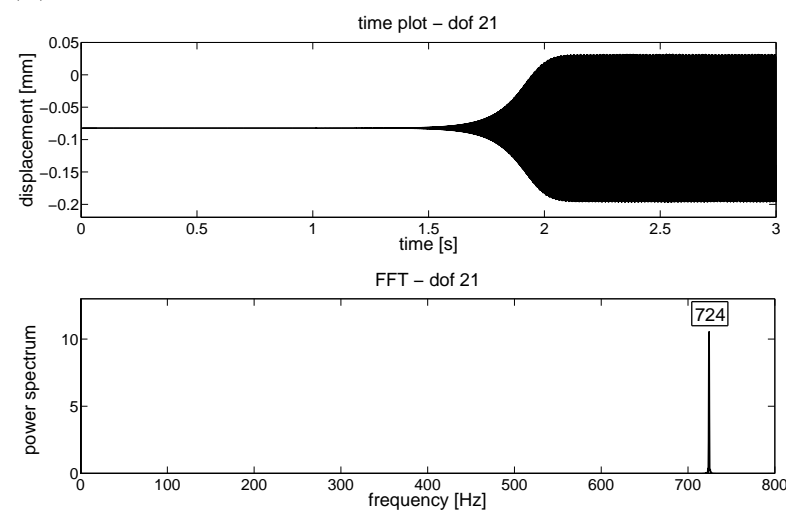

(b)
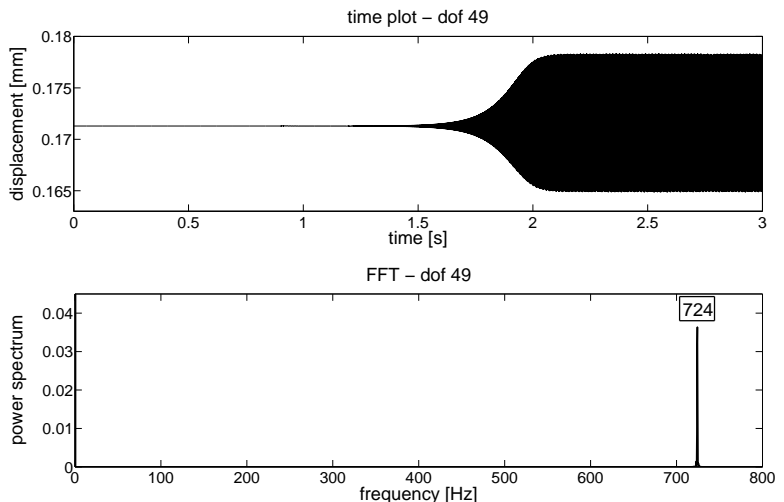

Figure 6: Squeal 2 time-history response and FFT: (a) dof 21, (b) dof 49

\subsubsection{Squeal 1}

The response of the Squeal 1 instability is presented in Fig. 7. On dof 21, the shape of the temporal behavior is quite similar to that of the Squeal 2 instability: no overshoot is obtained and the oscillation amplitudes remain constant after a short transient state. The first difference is the speed of development of the instability: the Squeal 1 instability develops faster than the Squeal 2 instability. However, it is known that the speed of increase depends directly on the real part of the instable eigenvalue, and may then be modified by the damping introduced for the simulation. The FFT shows that the response is made up of two components: the fundamental frequency at $320 \mathrm{~Hz}$, and its second harmonic at $640 \mathrm{~Hz}$. The stability analysis with damping gives an instable frequency of $316 \mathrm{~Hz}$. On dof 49, the time-plot reveals that the mean dynamical position of the steady-state oscillations is slightly different of the initial static position of the system. The power spectrum shows that the amplitude of the second harmonic is a lot larger than the amplitude of the fundamental component, unlike dof 21. The presence and size of the harmonic components can be seen as an indicator of the complexity of the nonlinear response.

(a)
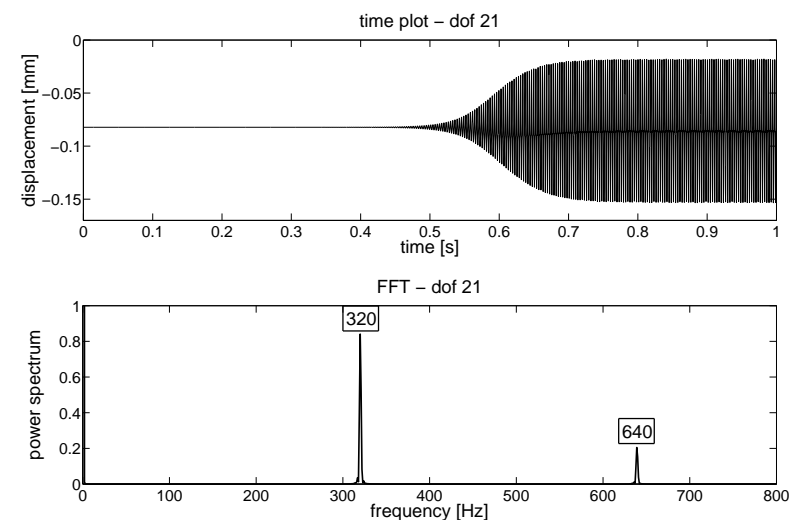

(b)
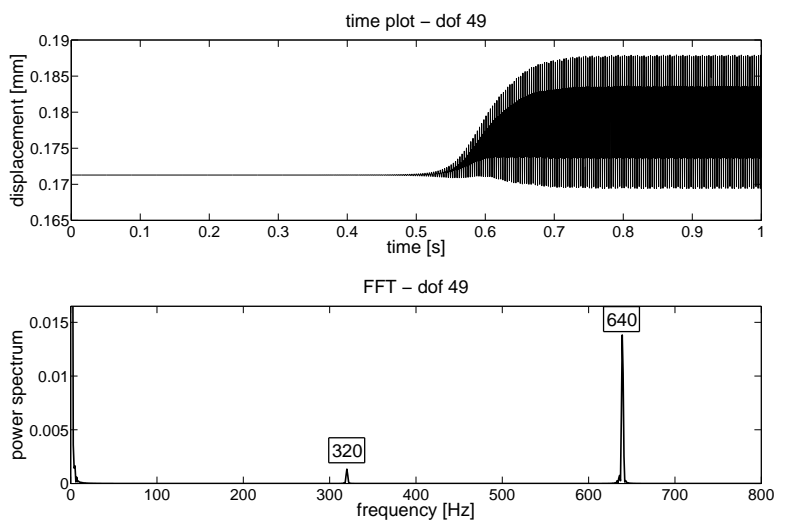

Figure 7: Squeal 1 time-history response and FFT: (a) dof 21, (b) dof 49

\subsubsection{Whirl 1}

The Whirl 1 instability is the first whirl-type instability presented. Its time-history response is given in Fig. 8. The first remark concerns the slowness of the development: at $t=12$ seconds, the stationary 
oscillations are not quite reached yet. This is linked to the small amount of negative damping present on the unstable mode, as can be seen in Fig. 4b. The time-plots of dofs 21 and 49 display fluctuations in oscillation amplitudes: they are more pronounced on dof 21 . This observation can be qualitatively correlated with the FFT power spectrum: as the response of dof 49 is almost monoharmonic at $48 \mathrm{~Hz}$, the FFT of dof 21 displays a second harmonic more than twice as large as the fundamental component. Moreover, the harmonics up to order 8 appear slightly on the power spectrum, the 7 -th one (at $336 \mathrm{~Hz})$ in particular is noticeable.

(a)

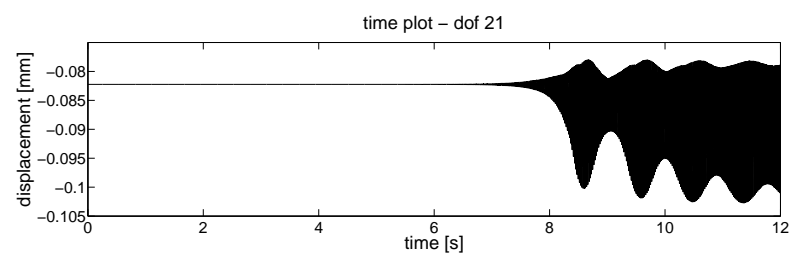

FFT - dof 21

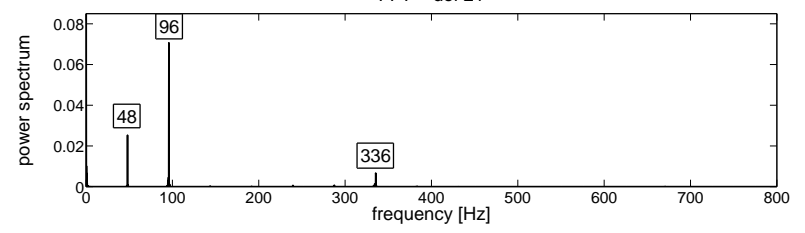

(b)

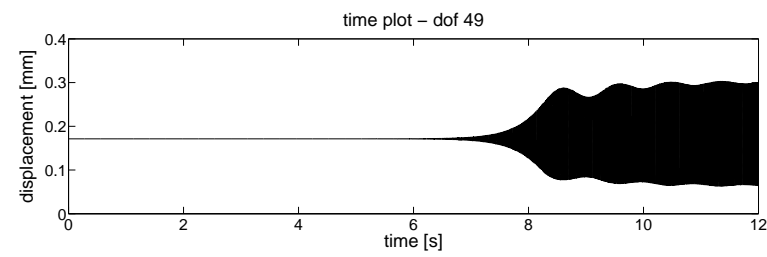

FFT - dof 49

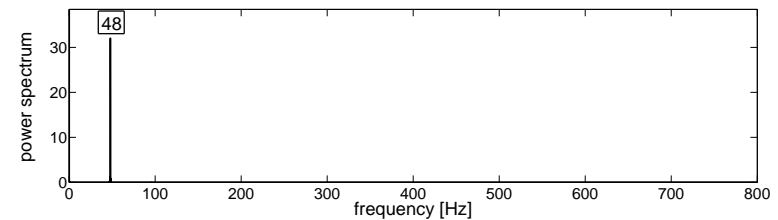

Figure 8: Whirl 1 time-history response and FFT: (a) dof 21, (b) dof 49

\subsubsection{Whirl 3}

For the Whirl 3 instability, the time-plots of Fig. 9 show significantly different behaviors whether seen on dof 21 or dof 49 . On the one hand, on dof 21 the vibration develops with a classically increase, quite similar to that observed for Squeal 1 or Squeal 2 instabilities: the fundamental frequency is given at 268 $\mathrm{Hz}$, and the second harmonic is hardly visible. On the other hand, the time-plot of dof 49 shows a lowfrequency component of high amplitude measured by FFT at $16 \mathrm{~Hz}$. This vibration appears abruptly at around $t=0.35$ second with the simultaneous abrupt variation of the mean dynamical position. This means that the brake is not vibrating around its initial static position (cf Fig. 10(a)). Although the oscillations of the low-frequency vibration decline fast (at $\mathrm{t}=1$ second they have almost disappeared), the mean position remains modified. A CWT is performed for the dof 49 time-history response for an in-depth study of what occurs. Thus Fig. 10(a) reveals that the whirl vibration with its second harmonic and the $16 \mathrm{~Hz}$ vibration seem to occur at the same time. To go into the analysis in greater depth, it appears that a mode of the structure is calculated by the stability analysis (cf Fig. 4) at around $16 \mathrm{~Hz}$. The movement of this mode is highly axial, as shown by its analytical shape given in Fig. 11. Thus the following explanation is proposed: when the whirl instability occurs, it causes a sudden variation of the position of the system. This jump mostly affects the axial dofs, such as dof 49 , and is then responsible for the excitation of an axial low-frequency mode of the structure. Without the jump of position, which acts as a Dirac excitation for the low-frequency mode, this vibration could not occur because it is not a coupling instability but an isolated structural mode. This clearly illustrates the complexity of the transient nonlinear behavior in brake systems, with the appearance and disappearance of new fundamental frequencies that cannot be foreseen by the stability analysis. Another interesting element is highlighted in Fig. 10(b). A CWT is performed around the jump of position for the fundamental whirl frequency. Simultaneous to the variation of position, the frequency of the vibration is also subjected to a quick shift of $17 \mathrm{~Hz}$ : as the instability appears at the beginning at around $285 \mathrm{~Hz}$ (which corresponds exactly to the frequency obtained by the stability analysis), the stationary frequency is measured at $268 \mathrm{~Hz}$. The change in the frequency may be seen as an effect of the change of the position, resulting from the modification of the equilibrium point. 
(a)


(b)
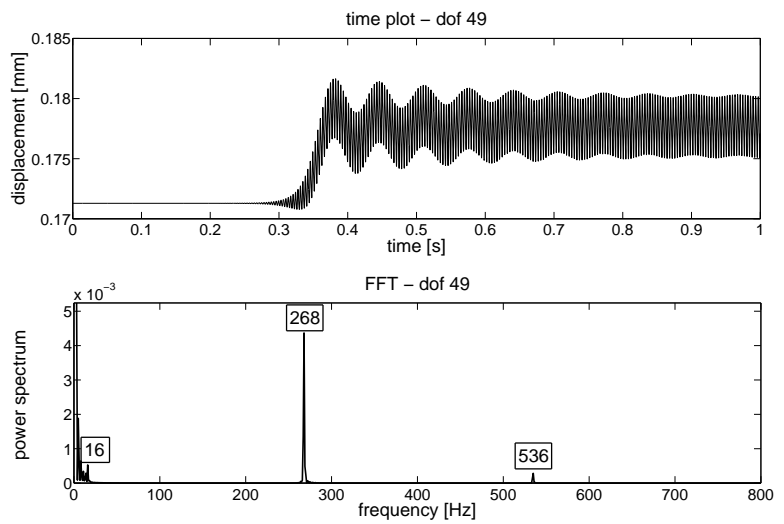

Figure 9: Whirl 3 time-history response and FFT: (a) dof 21, (b) dof 49

(a)


(b)
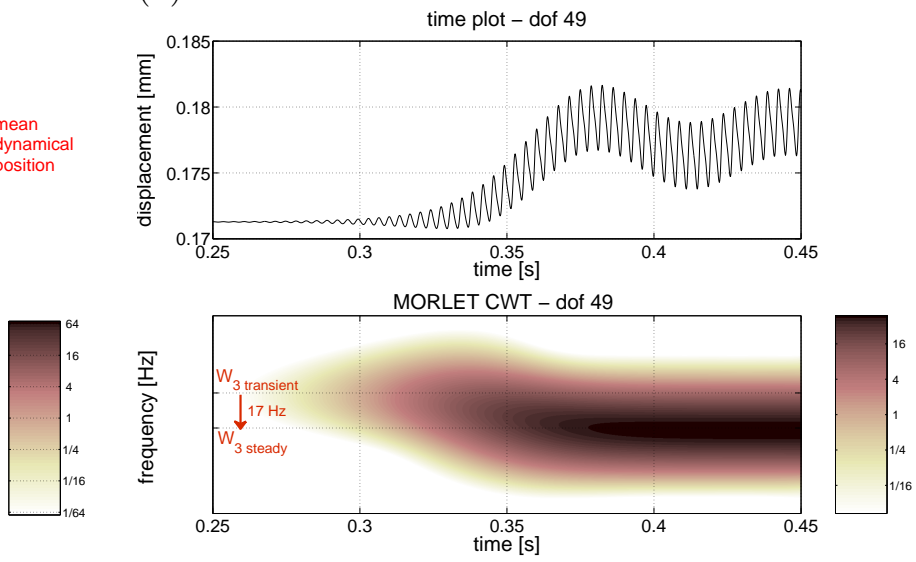

Figure 10: Whirl 3 time-history response and CWT: (a) global, (b) zoom

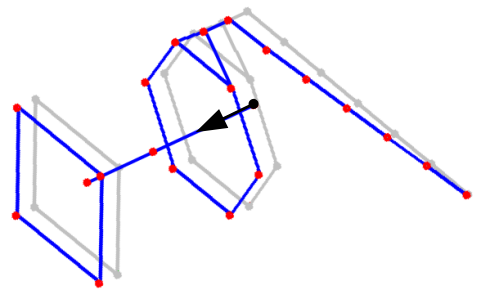

Figure 11: Analytical mode shape of the $16 \mathrm{~Hz}$ instability 


\subsubsection{Whirl 2}

Regarding the Whirl 2 time-history response illustrated in Figs. 12 and 13, its behavior is very close to that of the Whirl 3 instability. However, there is greater involvement of overharmonics, in particular the contribution of the 4-th harmonic (at $660 \mathrm{~Hz}$ ) is significant for the dof 21. It is interesting to observe that the jump of position (the difference between the mean dynamical position and the initial static position) is almost 8 times larger than that in the Whirl 3 case: $0.03 \mathrm{~mm}$ against $0.004 \mathrm{~mm}$. However, the effect on the frequency jump is the opposite: $3 \mathrm{~Hz}$ versus $17 \mathrm{~Hz}$. Thus, although the jump of position and the jump of frequency can be linked, the mechanisms are obviously not quite the same between these two instabilities. Moreover, the same low-frequency component at $16 \mathrm{~Hz}$ appears here, but much more significantly.

(a)
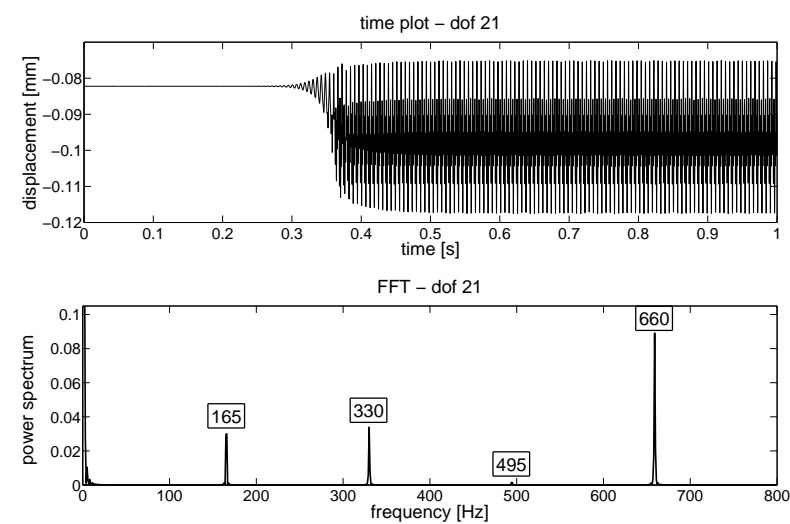

(b)
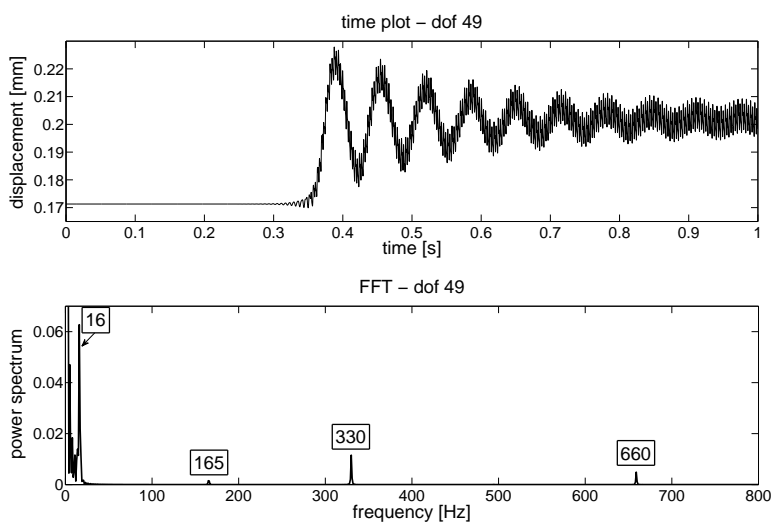

Figure 12: Whirl 2 time-history response and FFT: (a) dof 21, (b) dof 49

(a)


(b)
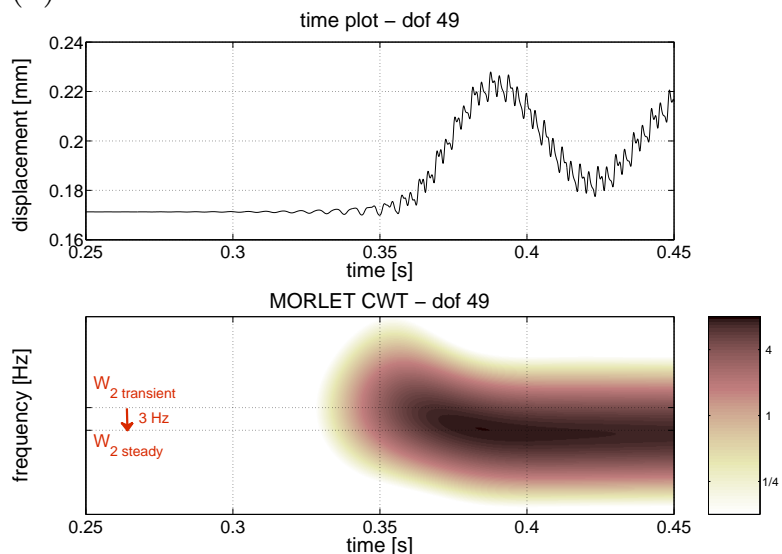

Figure 13: Whirl 2 time-history response and CWT: (a) global, (b) zoom

\subsubsection{Conclusions}

The main observation that may be expressed regarding the study of the five instabilities taken separately is that dynamical behavior can be rather complex, even in the case of single instability destabilization. Phenomena of variations in position and in frequency were highlighted with the help of CWT. Although the examples presented only show a variation in the frequency of less than $5 \%$, simulations, not presented in this study, carried out with other system parameters revealed variations in frequency up to about $20 \%$. Thus the 
usefullness of time-history computation is asserted: stability analysis can indeed be misleading with respect to the frequency of the instability. Transient nonlinear behavior also revealed the sometimes significant contribution of overharmonics up to a high order. Moreover, a complex mechanism of destabilization of a lowfrequency vibration was studied: an unexpected mode of the structure can be excited simultaneously with mode-coupling instability. In this case, the simulations have shown that the low-frequency vibration yields oscillations with an amplitude higher than that of the mode-coupling instability itself. This destabilization mechanism is very difficult to predict, making temporal integration necessary.

\subsection{Multi-instability behavior}

Single instability simulations revealed the rather complex dynamics of the brake in vibration. In this section, multi-instability simulations are performed. The aim is to examine how the system behaves when several instabilities due to friction are involved. 5 instabilities can be obtained, in line with the previous stability analysis. At least $\sum_{i=2}^{5} C_{5}^{i}=26$ simulations have to be performed in order to study all the combinations of $2,3,4$ and 5 instabilities. The most interesting ones are presented and analyzed in the following paragraphs.

\subsubsection{Whirl $3+$ Squeal 2}

The combination of Whirl 3 and Squeal 2 instabilities is examined. The time-plot and CWT are given in Fig. 14. The time-plot reveals slow growth: about 8 seconds are necessary to reach the steady-state oscillations. The behavior observed on dof 49 is interesting. At around 0.35 second, oscillations appear and a jump in position is noticed. This behavior is similar to that of the Whirl 3 instability in the stand-alone configuration. Between 1 and 4 seconds, steady-state oscillations seem to be obtained, but from around $\mathrm{t}=4$ seconds, the oscillations increase again. The CWT allows better understanding. The Whirl 3 vibration appears very quickly, with its second harmonic. The low-frequency mode at $16 \mathrm{~Hz}$ is destabilized at the same time, but does not appear on the figure because the CWT is only performed between 64 and $1024 \mathrm{~Hz}$. From $t=4$ seconds, the extra oscillations are due to the Squeal 2 instability. It may be noticed that the Whirl 3 vibration is not disrupted at all by the Squeal 2 vibration. The only interferences between the two instabilities take the form of frequency combinations at $\omega_{\text {squeal2 }}-\omega_{\text {whirl3 }}$ and $\omega_{\text {squeal2 }}+\omega_{\text {whirl3 }}$. However, their contribution is small.

(a)
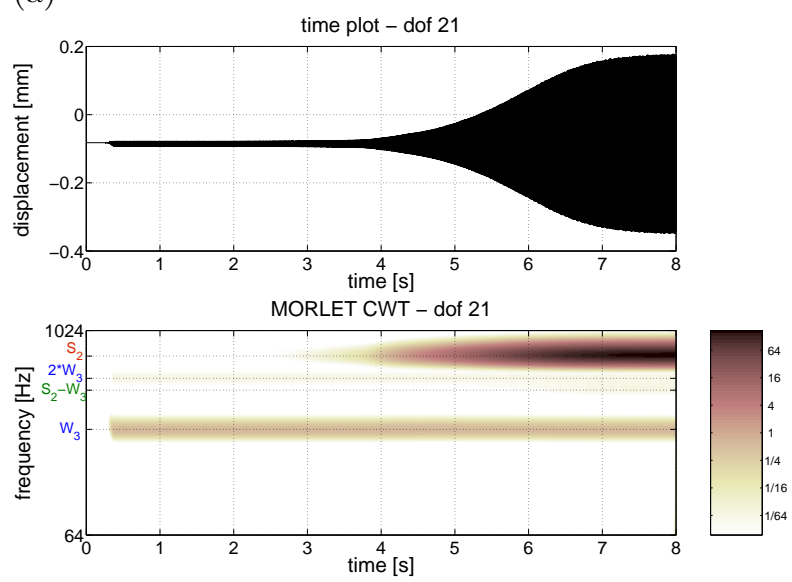

(b)
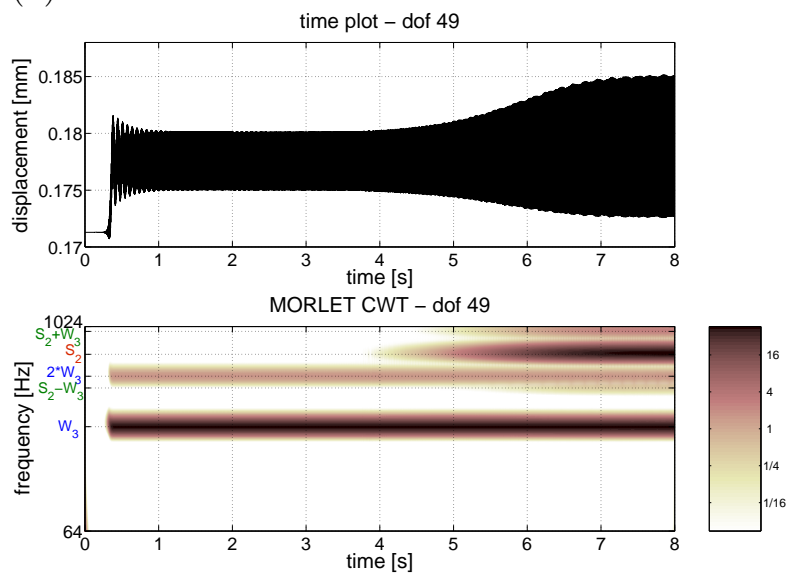

Figure 14: Mixing of Whirl 3 and Squeal 2, time-plot and CWT: (a) dof 21, (b) dof 49

\subsubsection{Whirl 2+ Whirl 3}

A different behavior is obtained when Whirl 2 and Whirl 3 instabilities are blended. As seen in Fig. 15(a), there the dynamics appear to comprise two steps: in the first one, a vibration develops, identified 
as the Whirl 3 vibration by using CWT, while in the second step at $t=0.45$ second Whirl 2 oscillations appear. The novelty compared to the previous case is that the two instabilities can not coexist at the same time: when the second vibration develops, it takes the place of the first one. The detailed CWT of Fig. 15(b) gives a view of all the frequencies between $128 \mathrm{~Hz}$ and $1024 \mathrm{~Hz}$ making up the signal. Are present, in the first part of the time-plot, Whirl 3 with its overharmonics $\left(2 \omega_{\text {whirl3 }}\right.$ and $\left.3 \omega_{\text {whirl3 }}\right)$, and in the second part, Whirl 2 with its overharmonics $\left(2 \omega_{\text {whirl2 }}, 3 \omega_{\text {whirl2 }}, 4 \omega_{\text {whirl2 }}\right.$ and $\left.5 \omega_{\text {whirl2 }}\right)$. In addition to this there are several frequency combinations, which involve fundamental or overharmonics frequencies of the two instabilities: $\omega_{\text {whirl3 }}+\omega_{\text {whirl2 }}, 2 \omega_{\text {whirl3 }}+\omega_{\text {whirl2 }}, 2 \omega_{\text {whirl3 }}-\omega_{\text {whirl2 }}$ and $3 \omega_{\text {whirl3 }}-\omega_{\text {whirl2 }}$ can be identified. These combinations only exist in the short temporal interval of the transition between the two instabilities. However, the contributions of the frequency components are not equal: the main ones are $\omega_{\text {whirl2 }}, \omega_{\text {whirl3 } 3}$, $2 \omega_{\text {whirl2 }}, \omega_{\text {whirl3 }}+\omega_{\text {whirl2 }}$ and $4 \omega_{\text {whirl2 }}$. This example is a good illustration of the complexity of the nonlinear transient dynamics: given two frequencies corresponding to two instabilities expected, the analysis of the time-history response highlights numerous frequencies that can be puzzling at first sight.

(a)
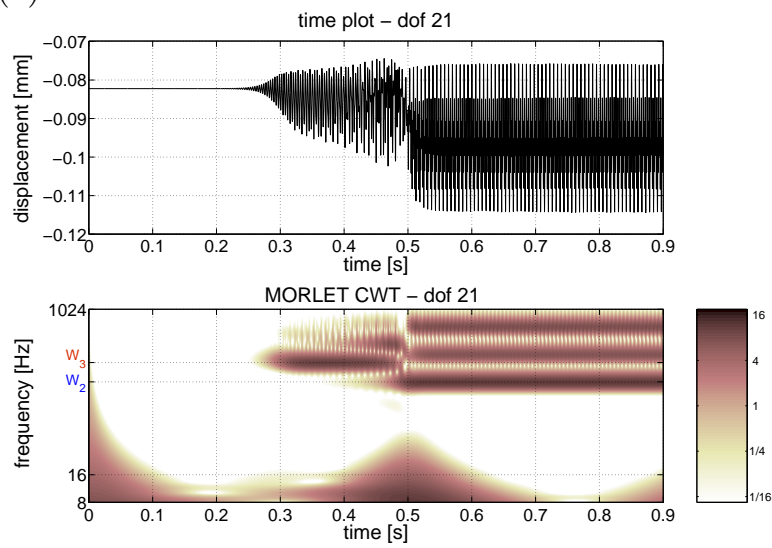

(b)

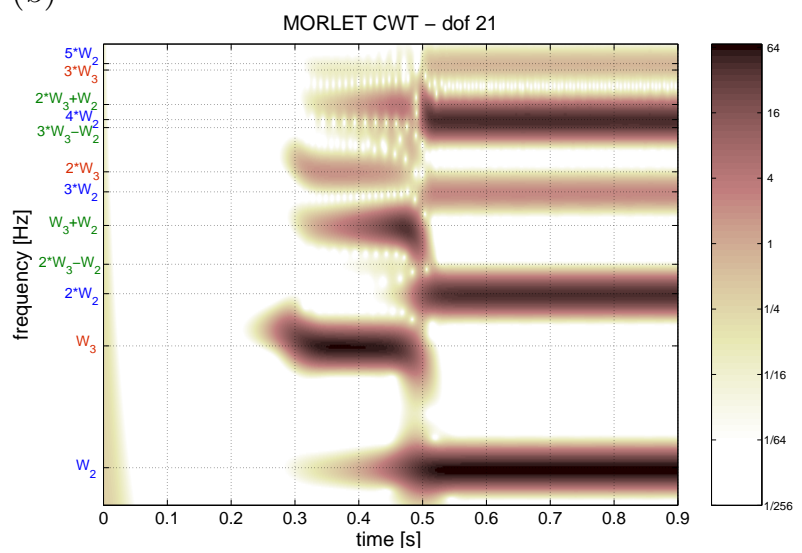

Figure 15: Mixing of Whirl 2 and Whirl 3: (a) time-plot and CWT, (b) detailed CWT

A further analysis is performed with a second simulation. The only different parameter is the damping introduced on the Whirl 2 modes which is slightly increased (from $4.0 \%$ to $4.5 \%$ ). In this case, the dynamics is quite similar but the moment of the transition between the two instabilities is altered as observed in Fig. 16. Since Whirl 2 is slightly more damped, it develops more slowly.



Figure 16: Second example of mixing of Whirl 2 and Whirl 3: time-plot and CWT 


\subsubsection{Whirl 2+ Squeal 1}

The Squeal 1 instability is very sensitive when combined with a Whirl 2 or Whirl 3 instability: either the whirl or squeal develops predominantly. Fig. 17 illustrates this point: the time-history responses of three configurations with slightly different damping introduced on the squeal instability $(1.0 \%, 1.5 \%$ and $2.0 \%)$ are represented. In case (a), only the Whirl 2 instability appears (with its harmonics of order 2,3 and 4), with low amplitude oscillations. The damping is reduced in case (b) to allow the squeal vibration to develop. The squeal vibration destabilizes as expected, with high levels of oscillation. The second harmonic also appears in the $64-1024 \mathrm{~Hz}$ range. What was not expected is the absence of whirl components in the response. In case (c), an intermediate damping is introduced in an attempt to obtain the interference of squeal with whirl. It is seen that squeal develops in the first part of the response in the form of a short burst, that then decreases when whirl increases. As in the two previous simulations, overharmonics $\left(2 \omega_{\text {whirl2 }}, 3 \omega_{\text {whirl2 }}\right.$, $4 \omega_{\text {whirl2 }}$ and $\left.2 \omega_{\text {squeal1 }}\right)$ are present, but several combinations can also be observed at $\omega_{\text {squeal1 }}+\omega_{\text {whirl2 }}$ and $2 \omega_{\text {squeal1 }}+\omega_{\text {whirl2 }}$ only during the transition between the two instabilities. In the steady-state oscillations, only the whirl components remain. Consequently, as with the combination of Whirl 2 and Whirl 3, Whirl 2 and Squeal 1 cannot coexist together for long. It is interesting to note that the two configurations (a) and (c) lead to the same steady-state oscillations, but have distinct transient behaviors.

(a)
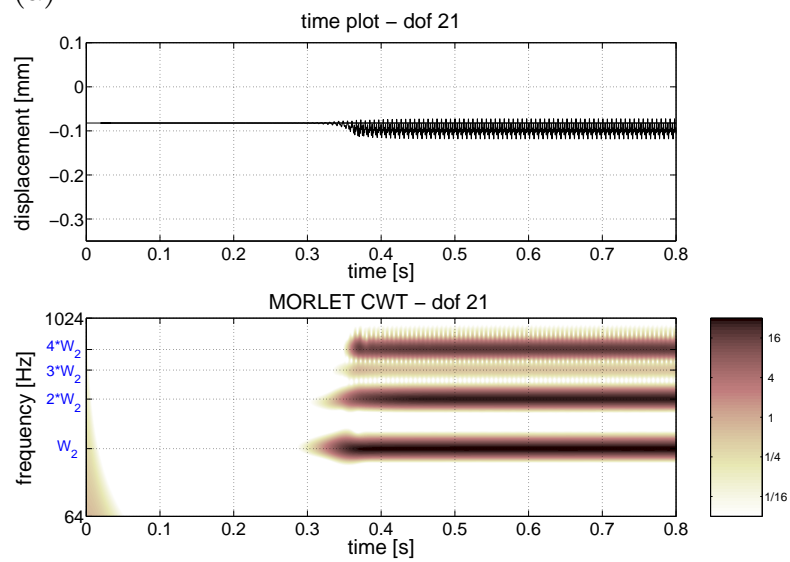

(c)
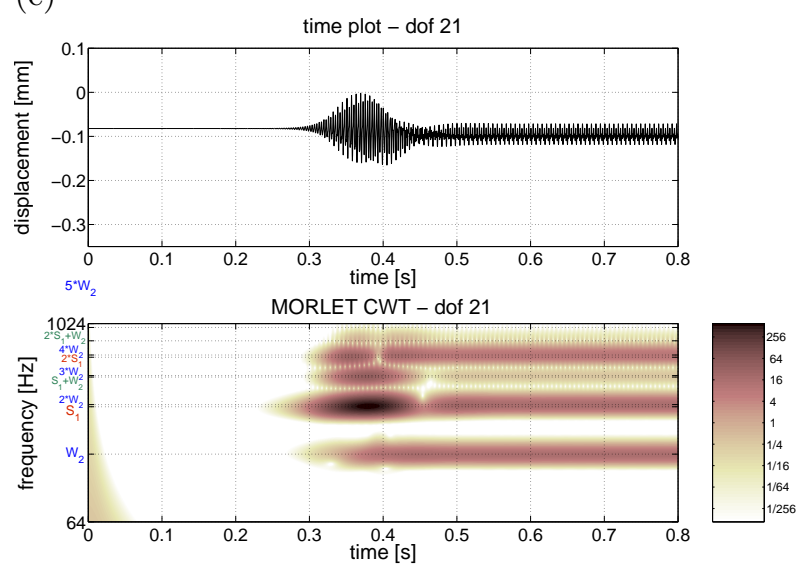

(b)
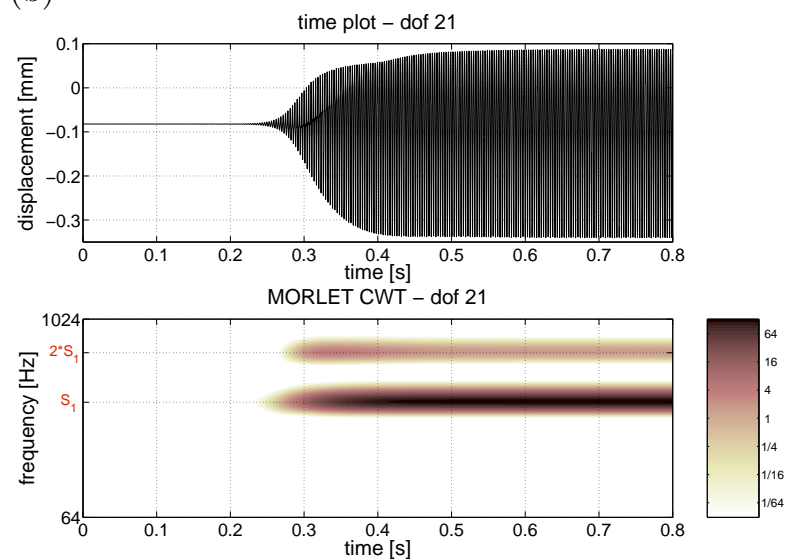

Figure 17: Mixing of Whirl 2 and Squeal 1 with different dampings on Squeal 1: (a) 2.0\%, (b) 1.0\%, (c) 1.5\% 


\subsubsection{Whirl 3+ Squeal 1}

As seen in the previous paragraph, Squeal 1 coexists with difficulty with Whirl 2 instability. The same behavior is obtained when Squeal 1 is mixed with Whirl 3. A configuration that allows destabilizing both instabilities is given in Fig. 18. The squeal burst phenomenon is also obtained, but the overshoot is less definite: the Whirl 3 oscillations remain predominant. The CWT computed between $32 \mathrm{~Hz}$ and $1024 \mathrm{~Hz}$ allows identifying the overharmonics of the two instabilities: $2 \omega_{\text {whirl3 }}, 3 \omega_{\text {whirl3 }}$ and $2 \omega_{\text {squeal1 }}$. The coexistence of the two instabilities seems to be somewhat easier than in the previous case, as the combinations are observed during the temporal interval of coexistence: $\omega_{\text {squeal1 }}-\omega_{\text {whirl3 }}, \omega_{\text {squeal1 }}+\omega_{\text {whirl3 }}$ and $2 \omega_{\text {whirl3 }}-$ $\omega_{\text {squeal1 }}$.
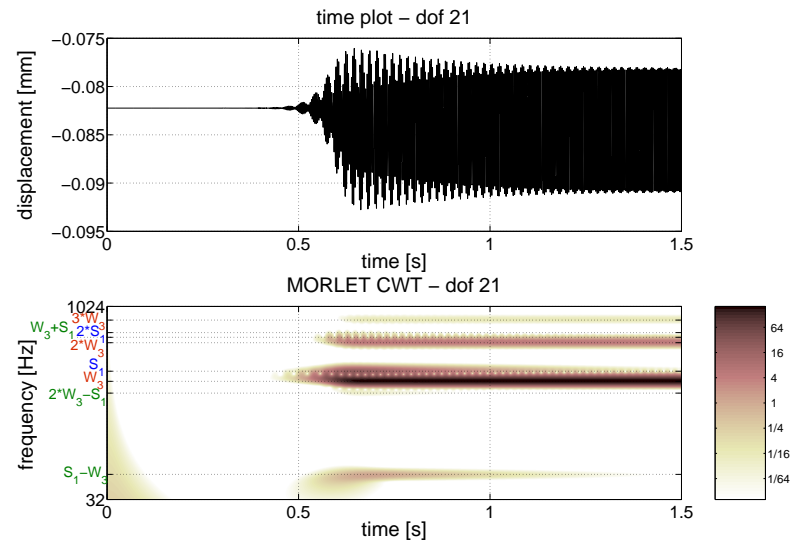

Figure 18: Combination of Whirl 3 and Squeal 1: time-plot and CWT

\subsubsection{Squeal $1+$ Squeal 2}

To complete the analysis of bi-instability behaviors, the two possible squeal instabilities are blended. Fig. 19 shows a predominance of Squeal 1 oscillations through harmonics $\omega_{\text {squeal1 }}$ and $2 \omega_{\text {squeal1 }}$. Squeal 2 appears after the destabilization of Squeal 1, but only lasts about 0.4 second. A small combination may be noticed at $\omega_{\text {squeal2 }}-\omega_{\text {squeal1 }}$. A particularity about the frequency of the Squeal 2 vibration can be noticed: although it develops at around $780 \mathrm{~Hz}$, the steady-state frequency is measured at $735 \mathrm{~Hz}$.
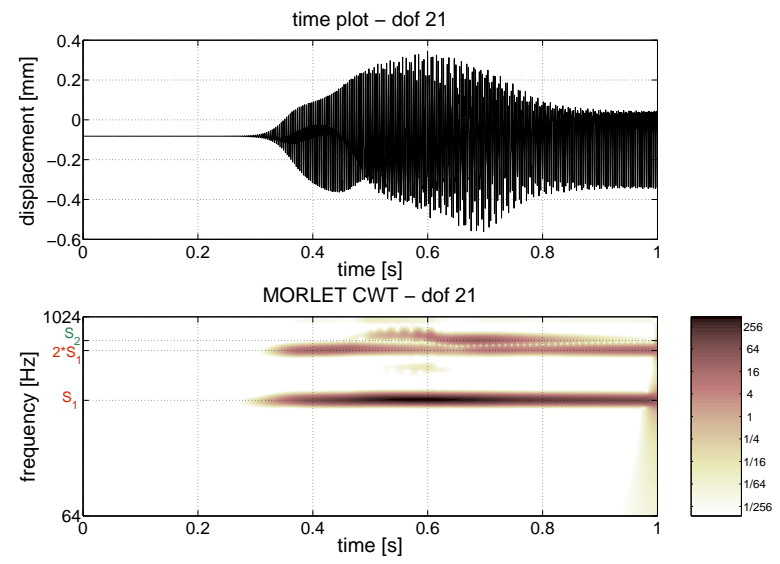

Figure 19: Combining Squeal 1 and Squeal 2: time-plot and CWT 


\subsubsection{Whirl 2+ Whirl 3+ Squeal 2}

Now that the mechanisms of the dynamical behavior of the brake when two instabilities are involved have been analyzed, the configurations with at least three instabilities are studied. The first case considers the combination of Whirl 2, Whirl 3 and Squeal 2. The previous paragraphs highlighted the incompatibility between Whirl 2 and Whirl 3. Concerning Squeal 2, it can react differently depending on whether it is combined with Whirl 2 or Whirl 3. It is not possible to predict behaviour when combining these three instabilities. An initial simulation is performed, the time-plot and CWT-plot are given in Fig. 20. Whirl 3 is the first instability to develop: its amplitude oscillations are small and the vibration only lasts about 0.5 second. Then the Whirl 2 instability appears, but since it is incompatible with the Whirl 3 vibration, the latter disappears. Whirl 2 continues while four very short bursts of Squeal 2 at very large amplitude emerge. The oscillations of Whirl 2 grow abruptly consecutive to the disapearance of Squeal: this is visible in particular on dof 49, where the destabilization of Whirl 2 is accompanied with a jump in the mean position and also with the $16 \mathrm{~Hz}$ vibration discussed in section 5.1.4. On the CWT plots only the fundamental frequencies are marked in view to improving visibility. However, the overharmonics and combinations can be noticed, the most prominent of which are $2 \omega_{\text {whirl2 }}, 3 \omega_{\text {whirl2 }}, 4 \omega_{\text {whirl2 }}, 2 \omega_{\text {whirl3 }}$ and $\omega_{\text {whirl2 }}+\omega_{\text {whirl3 }}$. In this example, the transient oscillations could be a lot more damaging for the system than the steady-state oscillations, composed of only Whirl 2 harmonics.

(a)
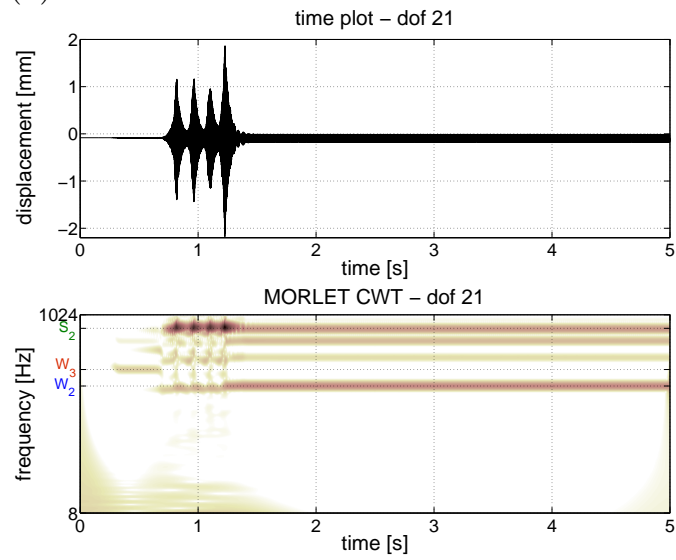

(b)
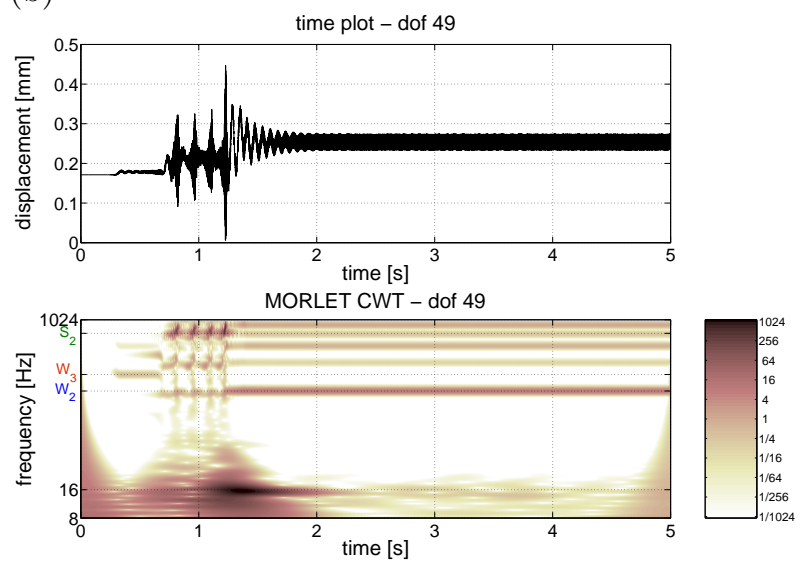

Figure 20: Mixing \#1 of Whirl 2, Whirl 3 and Squeal 2: (a) dof 21, (b) dof 49

A second simulation is performed with almost the same configuration: only a slight change in the damping is introduced on the Squeal 2 modes (from 1.0\% to 0.6\%). The result represented in Fig. 21 is surprising: the squeal bursts follow each another, with irregular intervals between two bursts. Unlike the previous simulation, no stationary regime seems to emerge: the computation of the transient response is inavoidable.

\subsubsection{Whirl 2+ Whirl 3 + Squeal $1+$ Squeal 2}

A final configuration where all five instabilities are destabilized is considered. This configuration is used for two simulations. The first one is presented in Fig. 22. Squeal 2 is the instability yielding the largest oscillations: it takes the form of a burst at the beginning of the simulation. Squeal 1 also appears to be destabilized in the transient regime. Regarding Whirl 2, its harmonics compose the stationary oscillations. Like Whirl 1, Whirl 3 does not appear. In fact, Whirl 1 hardly undergoes any destabilization when another instability appears. In section 5.1 it was shown that Whirl 1 develops very slowly, and in most cases Whirl 1 disappears if another instability appears. The time-plot of dof 49 shows the presence of the $16 \mathrm{~Hz}$ vibration, which appears with high amplitude oscillations simultaneously to the Whirl 2 vibration. The transition from Squeal 2 to Whirl 2 seems to occur due to the proximity of the fundamental frequency of Squeal 2 with the 4-th harmonic of Whirl 2. 
(a)
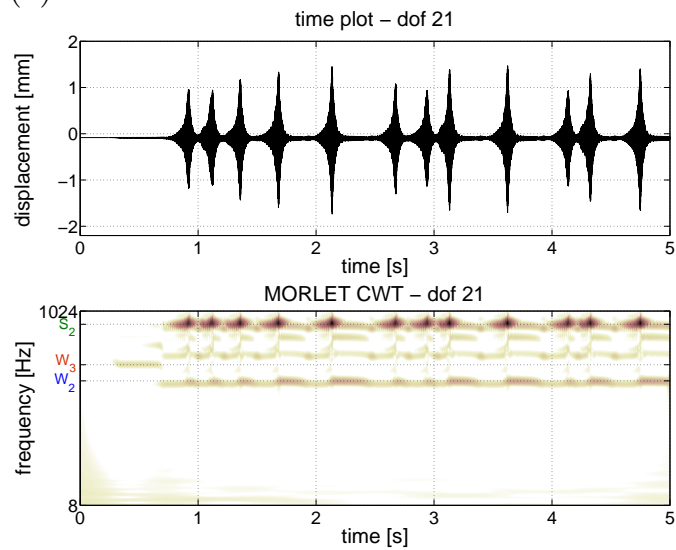

(b)



MORLET CWT - dof 49

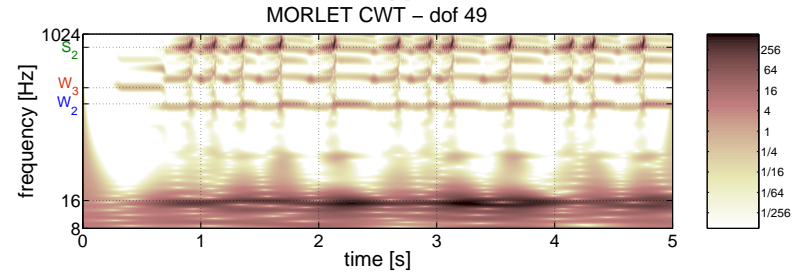

Figure 21: Mixing \#2 of Whirl 2, Whirl 3 and Squeal 2: (a) dof 21, (b) dof 49

(a)

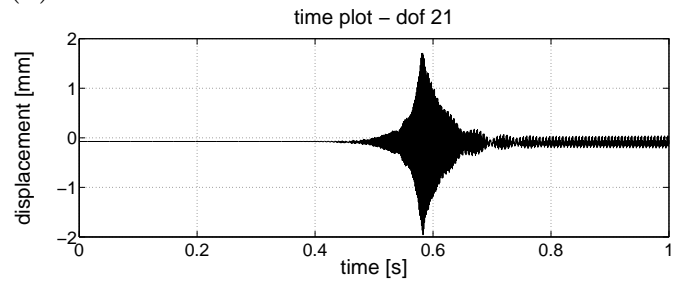

MORLET CWT - dof 21

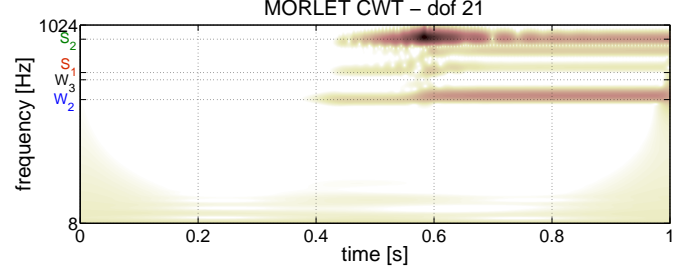

(b)

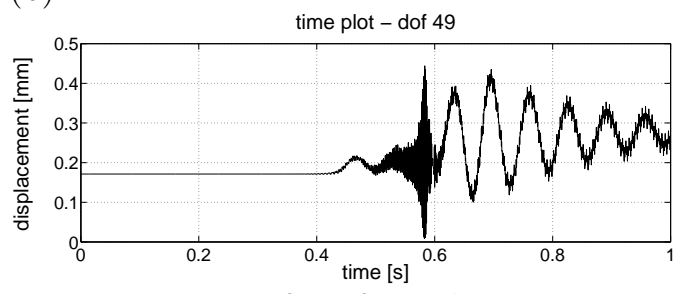

MORLET CWT - dof 49

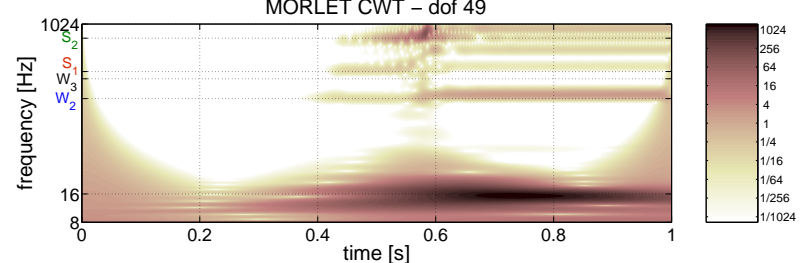

Figure 22: Mixing \#1 of Whirl 2, Whirl 3, Squeal 1 and Squeal 2: (a) dof 21, (b) dof 49 
The second simulation (cf Fig. 23) uses slightly different damping coefficients (cf Appendix B). Although the differences in the coefficients are small, the time-history responses are very dissimilar. In the first part, Whirl 3 and Squeal 1 are destabilized: the overharmonics of Whirl $\left(2 \omega_{\text {whirl3 }}\right)$ and Squeal $\left(2 \omega_{\text {squeal1 }}\right)$ are present and combinations also occur (mainly $\omega_{\text {squeal1 }}-\omega_{\text {whirl3 }}$ ). After the disappearance of the Squeal 1 vibration (which only lasts about 0.2 second), a transition occurs from Whirl 3 to Whirl 2 (through the combination $\left.\omega_{\text {whirl3 }}+\omega_{\text {whirl2 }}\right)$. Finally, Squeal 2 develops a little later, probably due to the 4 -th harmonic of Whirl 2, as in the previous simulation. The two simulations are similar in that Squeal 2 is the most critical in both cases. However, in one case it takes the form of a short burst with very high amplitude, while in the second case stationary oscillations remain at a more moderate amplitude. It can also be seen that only small changes in the damping configurations can lead to very different behaviors in both transient and steady-state regimes.

(a)

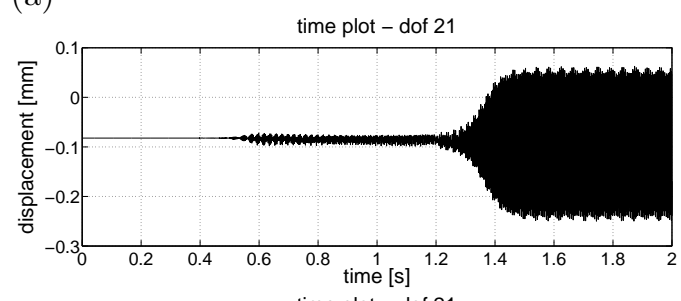

time plot - dof 21

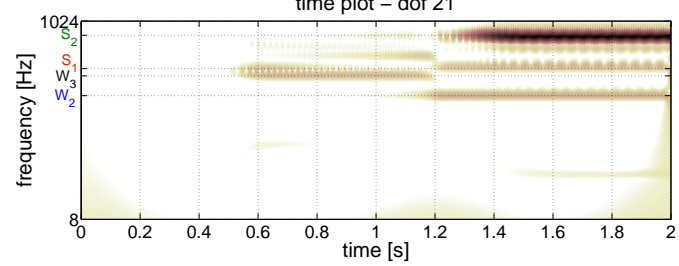

(b)
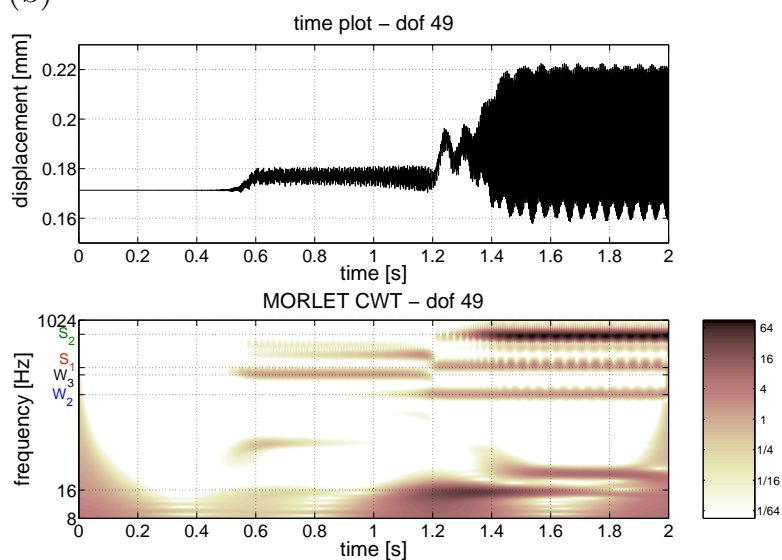

Figure 23: Mixing \#2 of Whirl 2, Whirl 3, Squeal 1 and Squeal 2: (a) dof 21, (b) dof 49

\subsubsection{Conclusions}

Time-history simulations with several types of mode-coupling instabilities have highlighted complicated dynamical mechanisms. The complexity seems to increase with the number of instabilities considered. When several instabilities are involved, only in very rare cases is the response of the system close to the sum of the responses obtained separately with each instability. Interactions often occur between the instabilities so that when several instabilities appear with their respective harmonics, along with interactions composed of combinations of frequencies, it can be difficult to interpret the spectral analysis despite the considerable aid provided by CWT. The results presented are very interesting because they make it possible to determine which instabilities can coexist with other instabilities, and which of the latter overwhelm the others. Thus it appears that two kinds of whirl instabilities cannot be simultaneously destabilized: when Whirl 2 and Whirl 3 are involved, then Whirl 3 is the first to develop, but quickly gives way to the Whirl 2 oscillations. As for Whirl 1, in most cases it is overwhelmed by any other instability. The combination of the two squeal instabilities leads to a predominance of Squeal 2. The most interesting cases concern the combination of squeal instability with a Whirl 2 or Whirl 3 instability. The analysis of Squeal shows that it tends to take the form of a very short burst at the beginning of braking, with very large oscillations.

Secondly, the simulations performed illustrate the complexity of the nonlinear transient response of braking systems. The same steady-state behavior can be obtained, but with a very different transient behavior, if an instability appears then disappears in the transient regime, as was shown in the examples given. In addition, it appears that multi-instability behaviors are very sensitive to the damping configuration. This sensitivity increases as a function of the number of instabilities involved. Behaviors with 4 coupling instabilities are very sensitive because variations in the damping coefficients can cause an instability to appear or disappear. However, for behavior with a single instability, variations in the damping coefficients 
will only modify the speed of increase of the oscillations and their amplitude. The experimental tests have only revealed behaviors with one and rarely two instabilities. Therefore the sensitivity of the responses observed by simulating multi-instability cases is not very disturbing, because these behaviors are obtained in very specific numerical cases that could hardly occur in reality.

\section{Summary}

In this paper, a nonlinear model for the simulation of mode-coupling instabilities induced by friction in aircraft braking systems is presented and used to investigate the transient behavior of the brake when vibrations occur. The temporal integration of the full set of nonlinear equations is computed with MATLAB ${ }^{\mathrm{TM}}$. The use of an analytical model with a small number of degrees of freedom is especially useful as the computation of several seconds of braking takes less than one hour on a desktop computer.

A stability analysis determines and identifies five mode-coupling instabilities. The time-history responses of single and multi-instability behaviors are obtained by modifying the damping configuration. They reveal complicated and interesting phenomena in the transient and stationary oscillations. Firstly, it appears that in a single instability configuration, stability and time-history can be correlated: if the stability analysis calculates an instability, the latter develops more or less quickly in the temporal response. However, the detailed analysis of the response with the help of CWT reveals complex phenomena. These are the participation of harmonics up to a high order, or abrupt variations of the mean position, in which case the system does not vibrate around its initial equilibrium point and the instable frequency can vary, or the sometimes substantial difference between the frequency obtained by the temporal integration and that given by the stability analysis, or else the destabilization mechanism of a stable single mode of the structure. Secondly, interactions between instabilities are investigated. In this case, when referring to the stability result, an expected instability does not systematically provide oscillations in the time-history response. Certain instabilities can overwhelm other instabilities. Complex mechanisms are seen to occur in multi-instability configurations: high amplitude transient oscillations can develop for a short duration that are potentially synonymous with damage caused to the mechanical system.

The study presented clearly demonstrates the need to compute the entire time-history response. It also highlights the role of damping: for a given set of brake parameters, a single variation of the damping configuration can produce very different system responses. In this context, an accurate estimation of the damping configuration is required to obtain good prediction of the brake's dynamical response. This is where the difficulty lies: measuring and simulating damping in real structures are probably among the most difficult issues in mechanics.

\section{Acknowledgments}

The authors would like to thank Messier-Bugatti for their permission to publish this work.

\section{Appendix A: nonlinear terms due to friction at the rotor-stator interface}

For a point $M$ of radial coordinates $(r, \theta)$, the normal relative displacement $\delta x$ is calculated by considering small displacements of the rotor and the stator:

$$
\begin{aligned}
& x_{\text {rotor }}(r, \theta)=x_{r}+r \sin \theta \sin \theta_{r}-r \cos \theta \sin \psi_{r} \approx x_{r}+r \theta_{r} \sin \theta-r \psi_{r} \cos \theta \\
& x_{\text {stator }}(r, \theta)=x_{s}+r \sin \theta \sin \theta_{s}-r \cos \theta \sin \psi_{s} \approx x_{s}+r \theta_{s} \sin \theta-r \psi_{s} \cos \theta
\end{aligned}
$$

that gives:

$$
\delta x(r, \theta)=x_{\text {stator }}(r, \theta)-x_{\text {rotor }}(r, \theta)=\left(x_{s}-x_{r}\right)+r \sin \theta\left(\theta_{s}-\theta_{r}\right)-r \cos \theta\left(\psi_{s}-\psi_{r}\right)
$$

where $x_{s}, x_{r}, \theta_{s}, \theta_{r}, \psi_{s}, \psi_{r}$ are respectively the stator and the rotor lateral displacements and rotations. 
Considering the cubic polynomial non-linear contact stress (Eq. (1)) and the Coulomb law (Eq. (3)), the normal force $F_{X}$, the tangential forces $F_{Y}, F_{Z}$, the brake torque $M_{X}$ and the yawing and pitching moments $M_{Y}, M_{Z}$ due to friction can then be expressed:

$$
\begin{aligned}
& F_{X}=\int_{0}^{2 \pi} \int_{R_{i}}^{R_{e}} N(r, \theta) r d r d \theta=\int_{0}^{2 \pi} \int_{R_{i}}^{R_{e}}\left(K_{1} \delta x(r, \theta)+K_{2} \delta x^{2}(r, \theta)+K_{3} \delta x^{3}(r, \theta)\right) r d r d \theta \\
& =K_{1} A_{2}\left(x_{s}-x_{r}\right)+K_{2}\left(A_{2}\left(x_{s}-x_{r}\right)^{2}+\frac{A_{4}}{4}\left(\theta_{s}-\theta_{r}\right)^{2}+\frac{A_{4}}{4}\left(\psi_{r}-\psi_{s}\right)^{2}\right) \\
& +K_{3}\left(A_{2}\left(x_{s}-x_{r}\right)^{3}+\frac{3 A_{4}}{4}\left(\theta_{s}-\theta_{r}\right)^{2}\left(x_{s}-x_{r}\right)+\frac{3 A_{4}}{4}\left(\psi_{s}-\psi_{r}\right)^{2}\left(x_{s}-x_{r}\right)\right) \\
& F_{Y}=-\int_{0}^{2 \pi} \int_{R_{i}}^{R_{e}} T(r, \theta) \sin \theta r d r d \theta=-\int_{0}^{2 \pi} \int_{R_{i}}^{R_{e}} \mu_{e q} N(r, \theta) \sin \theta r d r d \theta \\
& =-\mu_{e q}\left(K_{1} \frac{A_{3}}{3}\left(\theta_{s}-\theta_{r}\right)+K_{2} \frac{2 A_{3}}{3}\left(\theta_{s}-\theta_{r}\right)\left(x_{s}-x_{r}\right)\right. \\
& \left.+K_{3}\left(A_{3}\left(\theta_{s}-\theta_{r}\right)\left(x_{s}-x_{r}\right)^{2}+\frac{3 A_{5}}{20}\left(\theta_{s}-\theta_{r}\right)^{3}+\frac{3 A_{5}}{20}\left(\theta_{s}-\theta_{r}\right)\left(\psi_{s}-\psi_{r}\right)^{2}\right)\right) \\
& F_{Z}=\int_{0}^{2 \pi} \int_{R_{i}}^{R_{e}} T(r, \theta) \cos \theta r d r d \theta=\int_{0}^{2 \pi} \int_{R_{i}}^{R_{e}} \mu_{e q} N(r, \theta) \cos \theta r d r d \theta \\
& =-\mu_{e q}\left(K_{1} \frac{A_{3}}{3}\left(\psi_{s}-\psi_{r}\right)+K_{2} \frac{2 A_{3}}{3}\left(\psi_{s}-\psi_{r}\right)\left(x_{s}-x_{r}\right)\right. \\
& \left.+K_{3}\left(A_{3}\left(\psi_{s}-\psi_{r}\right)\left(x_{s}-x_{r}\right)^{2}+\frac{3 A_{5}}{20}\left(\psi_{s}-\psi_{r}\right)^{3}+\frac{3 A_{5}}{20}\left(\psi_{s}-\psi_{r}\right)\left(\theta_{s}-\theta_{r}\right)^{2}\right)\right) \\
& M_{X}=\int_{0}^{2 \pi} \int_{R_{i}}^{R_{e}} T(r, \theta) r^{2} d r d \theta=\int_{0}^{2 \pi} \int_{R_{i}}^{R_{e}} \mu_{e q} N(r, \theta) r^{2} d r d \theta \\
& =\mu_{e q}\left(K_{1} \frac{2 A_{3}}{3}\left(x_{s}-x_{r}\right)+K_{2}\left(\frac{2 A_{3}}{3}\left(x_{s}-x_{r}\right)^{2}+\frac{A_{5}}{5}\left(\theta_{s}-\theta_{r}\right)^{2}+\frac{A_{5}}{5}\left(\psi_{s}-\psi_{r}\right)^{2}\right)\right. \\
& \left.+K_{3}\left(\frac{2 A_{3}}{3}\left(x_{s}-x_{r}\right)^{3}+\frac{3 A_{5}}{5}\left(x_{s}-x_{r}\right)\left(\theta_{s}-\theta_{r}\right)^{2}+\frac{3 A_{5}}{5}\left(x_{s}-x_{r}\right)\left(\psi_{s}-\psi_{r}\right)^{2}\right)\right) \\
& M_{Y}=\int_{0}^{2 \pi} \int_{R_{i}}^{R_{e}} N(r, \theta) \sin \theta r^{2} d r d \theta \\
& =K_{1} \frac{A_{4}}{4}\left(\theta_{s}-\theta_{r}\right)+K_{2} \frac{A_{4}}{2}\left(\theta_{s}-\theta_{r}\right)\left(x_{s}-x_{r}\right) \\
& +K_{3}\left(\frac{3 A_{4}}{4}\left(\theta_{s}-\theta_{r}\right)\left(x_{s}-x_{r}\right)^{2}+\frac{3 A_{6}}{24}\left(\theta_{s}-\theta_{r}\right)^{3}+\frac{3 A_{6}}{24}\left(\theta_{s}-\theta_{r}\right)\left(\psi_{s}-\psi_{r}\right)^{2}\right) \\
& M_{Z}=-\int_{0}^{2 \pi} \int_{R_{i}}^{R_{e}} N(r, \theta) \cos \theta r^{2} d r d \theta \\
& =K_{1} \frac{A_{4}}{4}\left(\psi_{s}-\psi_{r}\right)+K_{2} \frac{A_{4}}{2}\left(\psi_{s}-\psi_{r}\right)\left(x_{s}-x_{r}\right) \\
& +K_{3}\left(\frac{3 A_{4}}{4}\left(\psi_{s}-\psi_{r}\right)\left(x_{s}-x_{r}\right)^{2} \frac{3 A_{6}}{24}\left(\psi_{s}-\psi_{r}\right)^{3}+\frac{3 A_{6}}{24}\left(\psi_{s}-\psi_{r}\right)\left(\theta_{s}-\theta_{r}\right)^{2}\right)
\end{aligned}
$$


with $A_{k}=\pi\left(R_{e}{ }^{k}-R_{i}{ }^{k}\right)$ for $\mathrm{k}=2$ to 6 and $R_{e}$ and $R_{i}$ respectively the outer and inner radius of the contact surface.

\section{Appendix B: damping configurations used for all simulations}

\begin{tabular}{|c|c|c|c|c|c|c|}
\hline Instabilities appearing & $\eta_{\text {whirl1 }}$ & $\eta_{\text {whirl2 }}$ & $\eta_{\text {whirl3 }}$ & $\eta_{\text {squeal1 }}$ & $\eta_{\text {squeal2 }}$ & $\eta_{\text {othermodes }}$ \\
\hline Squeal 2 & $5 \%$ & $10 \%$ & $35 \%$ & $5 \%$ & $\mathbf{0 . 9 \%}$ & $5 \%$ \\
\hline Squeal 1 & $5 \%$ & $10 \%$ & $35 \%$ & $\mathbf{2 . 5 \%}$ & $5 \%$ & $5 \%$ \\
\hline Whirl 1 & $\mathbf{0 . 0 1 \%}$ & $10 \%$ & $35 \%$ & $5 \%$ & $5 \%$ & $5 \%$ \\
\hline Whirl 3 & $5 \%$ & $10 \%$ & $\mathbf{3 0 \%}$ & $5 \%$ & $5 \%$ & $5 \%$ \\
\hline Whirl 2 & $5 \%$ & $\mathbf{4 \%}$ & $35 \%$ & $5 \%$ & $5 \%$ & $5 \%$ \\
\hline Whirl 3 and Squeal 2 & $5 \%$ & $10 \%$ & $\mathbf{3 0 \%}$ & $5 \%$ & $\mathbf{0 . 3 \%}$ & $5 \%$ \\
\hline Whirl 2 and Whirl 3 (case \#1) & $5 \%$ & $\mathbf{4 \%}$ & $\mathbf{3 0 \%}$ & $5 \%$ & $5 \%$ & $5 \%$ \\
\hline Whirl 2 and Squeal 1 (case \#1) & $5 \%$ & $\mathbf{4 . 5 \%}$ & $\mathbf{3 0 \%}$ & $5 \%$ & $5 \%$ & $5 \%$ \\
\hline Whirl 2 and Squeal 1 (case \#2) & $5 \%$ & $\mathbf{4 \%}$ & $35 \%$ & $\mathbf{2 \%}$ & $5 \%$ & $5 \%$ \\
\hline Whirl 2 and Squeal 1 (case \#3) & $5 \%$ & $\mathbf{4 \%}$ & $35 \%$ & $\mathbf{1 \%}$ & $5 \%$ & $5 \%$ \\
\hline Whirl 3 and Squeal 1 & $5 \%$ & $\mathbf{4 \%}$ & $35 \%$ & $\mathbf{1 . 5 \%}$ & $5 \%$ & $5 \%$ \\
\hline Squeal 1 and Squeal 2 & $5 \%$ & $10 \%$ & $35 \%$ & $\mathbf{1 \%}$ & $\mathbf{0 . 6 \%}$ & $5 \%$ \\
\hline Whirl 2, Whirl 3 and Squeal 2 case \#1) & $5 \%$ & $\mathbf{4 . 5 \%}$ & $\mathbf{3 0 \%}$ & $5 \%$ & $\mathbf{1 . 5 \%}$ & $5 \%$ \\
\hline Whirl 2, Whirl 3 and Squeal 2 (case \#2) & $5 \%$ & $\mathbf{4 . 5 \%}$ & $\mathbf{3 0 \%}$ & $5 \%$ & $\mathbf{1 \%}$ & $5 \%$ \\
\hline Whirl 2, Whirl 3, Squeal 1 and Squeal 2 case \#1) & $0.1 \%$ & $\mathbf{4 . 5 \%}$ & $\mathbf{3 0 \%}$ & $\mathbf{2 \%}$ & $\mathbf{0 . 6 \%}$ & $5 \%$ \\
\hline Whirl 2, Whirl 3, Squeal 1 and Squeal 2 case \#2) & $0.1 \%$ & $\mathbf{6 \%}$ & $\mathbf{3 0 \%}$ & $\mathbf{2 \%}$ & $\mathbf{1 \%}$ & $5 \%$ \\
\hline
\end{tabular}

Table 1: Damping configurations

\section{References}

[1] R.T. Spurr, A theory of brake squeal, Proceedings of the Institution of Mechanical Engineers 1 (1961-1962), 33-40.

[2] R.P. Jarvis, B. Mills, Vibrations induced by friction, Proceedings of the Institution of Mechanical Engineers 178 (1963), 847-857.

[3] H.R. Mills, Brake squeal, Technical Report No.9000B, Institution of Automobile Engineers, London (1938).

[4] R.A.C. Fosberry, Z. Holubecki, Disk brake squeal: Its mechanism and suppression, Technical Report No.1961/1, Motor Industry Research Association, Warwickshire, UK, 1961.

[5] H. Ouyang, J. Mottershead, M. Cartmell, M. Friswell, Friction-induced parametric resonances in discs: effect of a negative friction-velocity relationship, Journal of Sound and Vibration 209 (2) (1998), 251-264.

[6] S.S. Antoniou, A. Cameron, C.R. Gentle, The friction-speed relation from stick-slip data, Wear 36 (1976), 235-254.

[7] S.Y. Liu, M.A. Özbek, J.T. Gordon, A nonlinear model for aircraft brake squeal analysis. Part i: model description and solution methodology, AIAA paper AIAA-96-1251-CP (1996), 406-416.

[8] S.Y. Liu, M.A. Özbek, J.T. Gordon, A nonlinear model for aircraft brake squeal analysis. Part ii: stability analysis and parametric studies, AIAA paper AIAA-96-1252-CP (1996), 417-426.

[9] J.T. Gordon, A perturbation analysis of nonlinear squeal vibrations in aircraft braking systems, ASME Design Engineering Technical Conference, Sacramento, 1997.

[10] T.H. Travis, Nonlinear Transient Analysis of Aircraft Landing Gear Brake Whirl and Squeal, ASME Design Engineering Technical Conference 3, Vol.84-1, Part A (1995)

[11] L.B. Hagler, P.G. Reinhall, Friction induced vibrations in aircraft disk brakes, ASME Design Engineering Technical Conference, Sacramento, 1997.

[12] J-J. Sinou, O. Dereure, G-B. Mazet, F. Thouverez and L. Jézéquel, Friction induced vibration for an aircraft brake system. Part 1: Experimental approach and stability analysis, International Journal of Mechanical Sciences 48 (2006), 536-554.

[13] J-J. Sinou, F. Thouverez, L. Jézéquel, O. Dereure and G-B. Mazet, , Friction induced vibration for an aircraft brake system. Part 2: Non-linear dynamics, International Journal of Mechanical Sciences 48 (2006), 555-567.

[14] F. Chevillot, J-J. Sinou, G-B. Mazet, N. Hardouin and L. Jézéquel, The destabilization paradox applied to friction-induced vibrations in an aircraft braking system, Archive of Applied Mechanics 78 (2008), 949-963. 
[15] N. Coudeyras, J.-J. Sinou, S. Nacivet, A new treatment for predicting the self-excited vibrations of nonlinear systems with frictional interfaces: The Constrained Harmonic Balance Method, with application to disc brake squeal, Journal of Sound and Vibration 319 (2009), 1175-1199.

[16] A. Abubakar, H. Ouyang, Complex eigenvalue analysis and dynamic transient analysis in predicting disc brake squeal, International Journal of Vehicle Noise and Vibration 2 (2006), 143-155.

[17] F. Massi, L. Baillet, O. Giannini, A. Sestieri, Brake squeal: Linear and nonlinear numerical approaches, Mechanical Systems and Signal Processing 21 (2007), 2374-2393.

[18] D. Gabor, Theory of communication, IEEE Journal 93 (1946), 429-457.

[19] J. Morlet, Sampling theory and wave propagation, NATO ASI Series, F1, 233-261, Springer-Verlag, Berlin, 1983.

[20] S. Mallat, A Wavelet Tour of Signal Processing, Academic Press, New York, 1998.

[21] A. Louis, P. Maaß, A. Rieder, Wavelets, B.G. Teubner, Leipzig, 1994.

[22] C.K. Chui, An introduction to Wavelets, Academic Press, New York, 1992.

[23] R. Büssow, An algorithm for the continuous Morlet wavelet transform, Mechanical Systems and Signal Processing 21 (2007), 2970-2979.

[24] M.-N. Ta, J. Lardiès, Identification of weak nonlinearities on damping and stiffness by the continuous wavelet transform, Journal of Sound and Vibration 293 (2006), 16-37.

[25] P. Duffour, J. Woodhouse, Instability of systems with a frictional point contact. Part 1: basic modelling, Journal of Sound and Vibration 271 (2004), 365-390.

[26] F. Massi, O. Giannini, Effect of damping on the propensity of squeal instability: An experimental investigation, The Journal of the Acoustical Society of America 123-4(2008), 2017-2023.

[27] G. Fritz, J.-J. Sinou, J.-M. Duffal and L. Jézéquel Investigation of the relationship between damping and mode coupling patterns in case of brake squeal, Journal of Sound and Vibration 307, 3-5(2007), 591-609.

[28] J.-J. Sinou, L. Jézéquel, Mode coupling instability in friction-induced vibrations and its dependency on system parameters including damping, European Journal of Mechanics A/Solids 26 (2007), 106-122.

[29] B. Hervé, J.-J. Sinou, H. Mahé, L. Jézéquel, Analysis of squeal noise and mode coupling instabilities including damping and gyroscopic effects, European Journal of Mechanics A/Solids 27 (2008), 141-160. 